\title{
A Brief History of Cloud Application Architectures
}

\author{
Nane Kratzke \\ Lübeck University of Applied Sciences, Department of Electrical Engineering and Computer Science, \\ 23562 Lübeck, Germany; nane.kratzke@fh-luebeck.de
}

Received: 14 July 2018; Accepted: 27 July 2018; Published: 14 August 2018

Featured Application: This paper features system and software engineering use cases for large-scale (business) Cloud-native applications (e.g., Netflix, Twitter, Uber, Google Search). Such Cloud-native applications (CNA) provide web-scalability and independent deployability of their components and enable exponential user growth. Furthermore, migration and architecture transformation use cases of existing tiered and on-premise (business) applications are additionally of interest. Thus, questions of how existing and not cloud-ready applications are migratable into cloud environments are covered as well.

\begin{abstract}
This paper presents a review of cloud application architectures and its evolution. It reports observations being made during a research project that tackled the problem to transfer cloud applications between different cloud infrastructures. As a side effect, we learned a lot about commonalities and differences from plenty of different cloud applications which might be of value for cloud software engineers and architects. Throughout the research project, we analyzed industrial cloud standards, performed systematic mapping studies of cloud-native application-related research papers, did action research activities in cloud engineering projects, modeled a cloud application reference model, and performed software and domain-specific language engineering activities. Two primary (and sometimes overlooked) trends can be identified. First, cloud computing and its related application architecture evolution can be seen as a steady process to optimize resource utilization in cloud computing. Second, these resource utilization improvements resulted over time in an architectural evolution of how cloud applications are being built and deployed. A shift from monolithic service-oriented architectures (SOA), via independently deployable microservices towards so-called serverless architectures, is observable. In particular, serverless architectures are more decentralized and distributed, and make more intentional use of separately provided services. In other words, a decentralizing trend in cloud application architectures is observable that emphasizes decentralized architectures known from former peer-to-peer based approaches. This is astonishing because, with the rise of cloud computing (and its centralized service provisioning concept), the research interest in peer-to-peer based approaches (and its decentralizing philosophy) decreased. However, this seems to change. Cloud computing could head into the future of more decentralized and more meshed services.
\end{abstract}

Keywords: cloud computing; service-oriented architecture; SOA; cloud-native; serverless; microservice; container; unikernel; distributed cloud; P2P; service-to-service; service-mesh

\section{Introduction}

Even tiny companies can generate enormous economic growth and business value by providing cloud-based services or applications-Instagram, Uber, WhatsApp, NetFlix, and Twitter-and many astonishing small companies (if we relate the modest headcount of these companies in their founding days to their noteworthy economical impact) whose services are frequently used. However, 
even a fast-growing start-up business model should have its long-term consequences and dependencies in mind. A lot of these companies rely on public cloud infrastructures-currently often provided by Amazon Web Services (AWS). However, will AWS still be the leading and dominating cloud service provider in 20 years? IT history is full of examples of large and well-known companies failing and (almost) dieing: Atari, America Online (AOL), Compaq, Hewlett Packard, Palm, and Yahoo. Even Microsoft-still a prospering company-is no longer the dominating software company that it used to be in the 1990s and 2000s. Microsoft is even a good example of a company that has evolved and transformed into a cloud service provider. This might be because cloud providers are becoming more and more critical for national economies. Cloud providers run a significant amount of mission-critical business software for companies that no longer operate their own data-centers. Moreover, it is very often economically reasonable if workloads have a high peak-to-average ratio [1]. Thus, cloud providers might become (or even are) a too-big-to-fail company category that seems to become equally important for national economies like banks, financial institutions, electricity suppliers, public transport systems. Although essential for national economies, these financial, energy, or transport providers provide just replaceable goods or services—commodities. However, the cloud computing domain is still different here. Although cloud services could be standardized commodities, they are mostly not. Once a cloud-hosted application or service is deployed to a specific cloud infrastructure, it is often inherently bound to that infrastructure due to non-obvious technological bindings. A transfer to another cloud infrastructure is very often a time-consuming and expensive one-time exercise. A good real-world example here is Instagram. After being bought by Facebook, it took over a year for the Instagram engineering team to find and establish a solution for the transfer of all its services from AWS to Facebook data centers. Although no downtimes were planned, noteworthy outages occurred during that period.

The NIST definition of cloud computing defines three basic and well-accepted service categories [2]: Infrastructure as a Service (IaaS), Platform as a Service (PaaS), and Software as a Sevice (SaaS). IaaS provides maximum flexibility for arbitrary consumer-created software but hides almost no operation complexity of the application (just of the infrastructure). SaaS on the opposite hides operation complexity almost entirely but is too limited for many use cases involving consumer-created software. PaaS is somehow a compromise enabling the operation of consumer-created software with a convenient operation complexity but at the cost of following resource efficient application architectures and accepting to some degree lock-in situations resulting from the platform.

Throughout a project called CloudTRANSIT, we searched intensively for solutions to overcome this "cloud lock-in" - to make cloud computing an actual commodity. We developed and evaluated a cloud application transferability concept that has prototype status but already works for approximately $70 \%$ of the current cloud market, and that can be extended for the rest of the market share [3]. However, what is essential: we learned some core insights from our action research with practitioners:

1. Practitioners prefer to transfer platforms (and not applications).

2. Practitioners want to have the choice between platforms.

3. Practitioners prefer declarative and cybernetic (auto-adjusting) instead of workflow-based (imperative) deployment and orchestration approaches.

4. Practitioners are forced to make efficient use of cloud resources because more and more systems are migrated to cloud infrastructures causing steadily increasing bills.

5. Practitioners rate pragmatism of solutions much higher than full feature coverage of cloud platforms and infrastructures.

All these points influence ulteriorly how practitioners nowadays construct cloud application architectures that are intentionally designed for the cloud. This paper investigates the observable evolution of cloud application architectures over the last decade. 


\section{Methodology and Outline of This Paper}

Figure 1 presents the research methodology for this paper. The remainder of this paper follows this structure. Section 3 presents an overview of the research project CloudTRANSIT that build the foundation of our problem awareness of cloud application architectures. The project CloudTRANSIT intentionally tackled the cloud lock-in problem of cloud-native applications and analyzed how cloud-applications can be transferred between different cloud infrastructures at runtime without downtime. From several researchers as well as reviewer feedback, we get to know that the insights we learned about cloud architectures merely as a side-effect might be of general interest for the cloud computing research and engineering community.

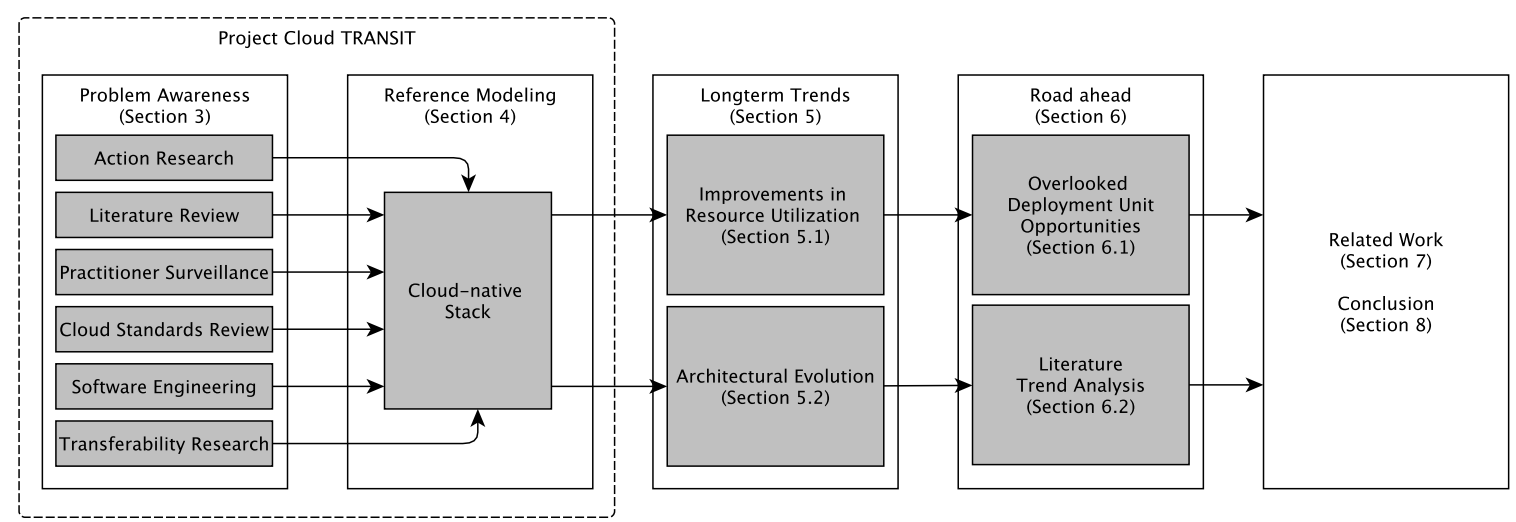

Figure 1. Research methodology.

One thing we learned was the fact that cloud-native applications-although they are all different-follow some common architectural patterns that we could exploit for transferability. Section 4 presents a reference model that structures such observable commonalities of cloud application architectures. Based on that insight, the obvious question that arises is what long-term trends exist that influence current shapes of cloud application architectures? Section 5 will investigate such observable long-term trends. In particular, we will investigate the resource utilization evolution in Section 5.1 and the architectural evolution in Section 5.2 and ends to some degree the observable status quo. However, the question is whether these long-term trends will go on in the future and can they be used for forecasts? Although forecasts are tricky in general and our research has not invented a crystal ball, Section 6 will take a look on the road ahead mainly by extrapolating these identified trends. Some aspects can be derived from the observed long-term-trends regarding optimization of resource efficiency in Section 6.1 and architectural changes by a Scopus based literature trend analysis in Section 6.2. Apparently, this paper is not the only one reflecting and analyzing cloud application architecture approaches, and the reader should take related work in Section 7 into account as well. Finally, we look at our brief history of cloud architectures and long-term trends. Assuming that these long-term trends will go on in the future for a while, we draw some conclusions on the road ahead in Section 8 .

\section{Problem Awareness (from the Research Project Cloud TRANSIT)}

Our problem awareness results mainly from the conducted research project CloudTRANSIT. This project dealt with the question of how to transfer cloud applications and services at runtime without downtime across cloud infrastructures from different public and private cloud service providers to tackle the existing and growing problem of vendor lock-in in cloud computing. Throughout the project, we published more than 20 research papers. However, the intent of this paper is not to summarize these papers. The interested reader is referred to the corresponding technical report [3] that provides an integrated view of these outcomes. 
This paper strives to take a step back and review the observed state of the art related to how cloud-based systems are being built today and how they might be built tomorrow. It might be of interest for the reader to get an impression of how the foundation for these insights has been derived by understanding the mentioned research project.

The project analyzed commonalities of existing public and private cloud infrastructures via a review of industrial cloud standards and cloud applications via a systematic mapping study of cloud-native application-related research [4]. Action research projects with practitioners accompanied this review. Latest evolutions of cloud standards and cloud engineering trends (like containerization) were used to derive a reference model that guided the development of a pragmatic cloud-transferability solution. We evaluated this reference model using a concrete project from our action research activities [5]. This solution intentionally separated the infrastructure-agnostic operation of elastic container platforms (like Swarm, Kubernetes, Mesos/Marathon, etc.) via a multi-cloud-scaler and the platform-agnostic definition of cloud-native applications and services via an unified cloud application modeling language. Both components are independent but complementary and provide a solution to operate elastic (container) platforms in an infrastructure-agnostic, secure, transferable, and elastic way. This multi-cloud-scaler is described in [6,7]. Additionally, we had to find a solution to describe cloud applications in a unified format. This format can be transformed into platform-specific definition formats like Swarm compose, Kubernetes manifest files, and more. This Unified Cloud Application Modeling Language (UCAML) is explained in [8,9]. Both approaches mutually influenced each other and therefore have been evaluated in parallel by deploying and transferring several cloud reference applications [10] at runtime [7,9]. This solution supports the public cloud infrastructures of AWS, Google Compute Engine (GCE), and Azure and open source infrastructure OpenStack. It covers approximately $70 \%$ of the current cloud market. Because the solution can be extended with cloud infrastructure drivers, the rest of the market share can also be supported by additional drivers of moderate complexity.

However, what is even more essential: We learned some core insights about cloud application architectures in general by asking the question of how to transfer this kind of applications without touching their application architectures. Let us investigate this in the following Section 4.

\section{Reference Modeling-How Cloud Applications Look}

Almost all cloud system engineers focus on a common problem. The core components of their distributed and cloud-based systems like virtualized server instances and basic networking and storage can be deployed using commodity services. However, further services- that are needed to integrate these virtualized resources in an elastic, scalable, and pragmatic manner-are often not considered in standards. Services like load balancing, auto-scaling or message queuing systems are needed to design an elastic and scalable cloud-native system on almost every cloud service infrastructure. Some standards like AMQP [11] for messaging (dating back almost to the pre-cloud era) exist. However, mainly these integrating and "gluing" service types—that are crucial for almost every cloud application on a higher cloud maturity level (see Table 1) —are often not provided in a standardized manner by cloud providers [12]. It seems that all public cloud service providers try to stimulate cloud customers to use their non-commodity convenience service "interpretations" to bind them to their infrastructures and higher-level service portfolios.

Furthermore, according to an analysis we performed in 2016 [13], the percentage of these commodity service categories that are considered in standards like CIMI [14], OCCI [15,16], CDMI [17], OVF [18], OCI [19], TOSCA [20] is even decreasing over the years. This mainly has to do with the fact that new cloud service categories are released faster than standardization authorities can standardize existing service categories. Figure 2 shows this effect by the example of AWS over the years. This is how mainly vendor lock-in emerges in cloud computing. For a more detailed discussion, we refer to $[5,13,21]$. 
Table 1. Cloud Application Maturity Model, adapted from OPEN DATA CENTER ALLIANCE [22].

\begin{tabular}{ccl}
\hline Level & Maturity & Criteria \\
\hline 3 & $\begin{array}{c}\text { Cloud } \\
\text { native }\end{array}$ & $\begin{array}{c}\text { - Transferable across infrastructure providers at } \\
\text { runtime and without interruption of service. } \\
\text { - Automatically scale out/in based on stimuli. }\end{array}$ \\
\hline 2 & $\begin{array}{c}\text { Cloud } \\
\text { resilient }\end{array}$ & $\begin{array}{l}\text { - State is isolated in a minimum of services. } \\
\text { - Unaffected by dependent service failures. } \\
\text { - Infrastructure agnostic. }\end{array}$ \\
\hline 1 & $\begin{array}{c}\text { Cloud } \\
\text { friendly }\end{array}$ & $\begin{array}{l}\text { - Composed of loosely coupled services. } \\
\text { - Services are discoverable by name. } \\
\end{array}$ \\
& & $\begin{array}{l}\text { - Components are designed to cloud patterns. } \\
\text { - Compute and storage are separated. }\end{array}$ \\
\hline 0 & Cloud & - Operated on virtualized infrastructure. \\
& ready & - Instantiateable from image or script. \\
\hline
\end{tabular}

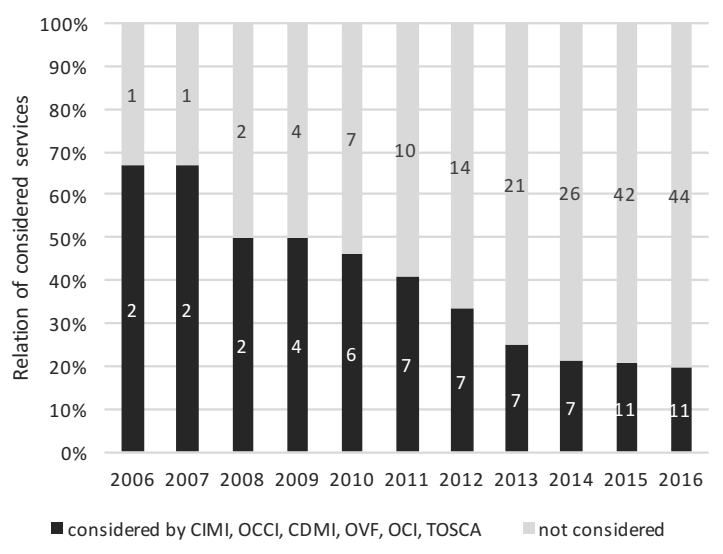

Figure 2. Decrease of standard coverage over years (by example of AWS).

Therefore, all reviewed cloud standards focus on a minimal but necessary subset of popular cloud services: compute nodes (virtual machines), storage (file, block, object), and (virtual private) networking. Standardized deployment approaches like TOSCA are defined mainly against this commodity infrastructure level of abstraction. These kinds of services are often subsumed as IaaS and build the foundation of cloud services and therefore cloud-native applications. All other service categories might foster vendor lock-in situations. This might sound disillusioning. In consequence, many cloud engineering teams follow the basic idea that a cloud-native application stack should be only using a minimal subset of well-standardized IaaS services as founding building blocks. Because existing cloud standards cover only specific cloud service categories (mainly the IaaS level) and do not show an integrated point of view, a more integrated reference model that takes best practices of practitioners into account would be helpful.

Very often, cloud computing is investigated from a service model point of view (IaaS, PaaS, SaaS), a deployment point of view (private, public, hybrid, community cloud) [2]. Alternatively, one can look from an actor point of view (provider, consumer, auditor, broker, carrier) or a functional point of view (service deployment, service orchestration, service management, security, privacy) as it is done by [23]. Points of view are particularly useful to split problems into concise parts. However, the viewpoints mentioned above might be common in cloud computing and useful from a service provider point of view but not from cloud-native application engineering point of view. From an engineering point of view, it seems more useful to have views on technology levels involved and applied in cloud-native application engineering. Practitioner models do this often. 
By using the insights from our systematic mapping study [24] and our review of cloud standards [5], we compiled a reference model of cloud-native applications. This layered reference model is shown and explained in Figure 3. The basic idea of this reference model is to use only a small subset of well-standardized IaaS services as founding building blocks (Layer 1). Four primary viewpoints form the overall shape of this model.

1. Infrastructure provisioning: This is a viewpoint being familiar for engineers working on the infrastructure level and how IaaS is understood. IaaS deals with the deployment of separate compute nodes for a cloud consumer. It is up to the cloud consumer what he does with these isolated nodes (even if there are hundreds of them).

2. Clustered elastic platforms: This is a viewpoint being familiar for engineers who are dealing with horizontal scalability across nodes. Clusters are a concept to handle many Layer 1 nodes as one logical compute node (a cluster). Such kind of technologies is often the technological backbone for portable cloud runtime environments because they are hiding complexity (of hundreds or thousands of single nodes) appropriately. Additionally, this layer realizes the foundation to define services and applications without reference to particular cloud services, cloud platforms or cloud infrastructures. Thus, it provides a foundation to avoid vendor lock-in.

3. Service composing: This is a viewpoint familiar for application engineers dealing with Web services in service-oriented architectures (SOA). These (micro)-services operate on a Layer 2 cloud runtime platform (like Kubernetes, Mesos, Swarm, Nomad, and so on). Thus, the complex orchestration and scaling of these services are abstracted and delegated to a cluster (cloud runtime environment) on Layer 2.

4. Application: This is a viewpoint being familiar for end-users of cloud services (or cloud-native applications). These cloud services are composed of smaller cloud Layer 3 services being operated on clusters formed of single compute and storage nodes.

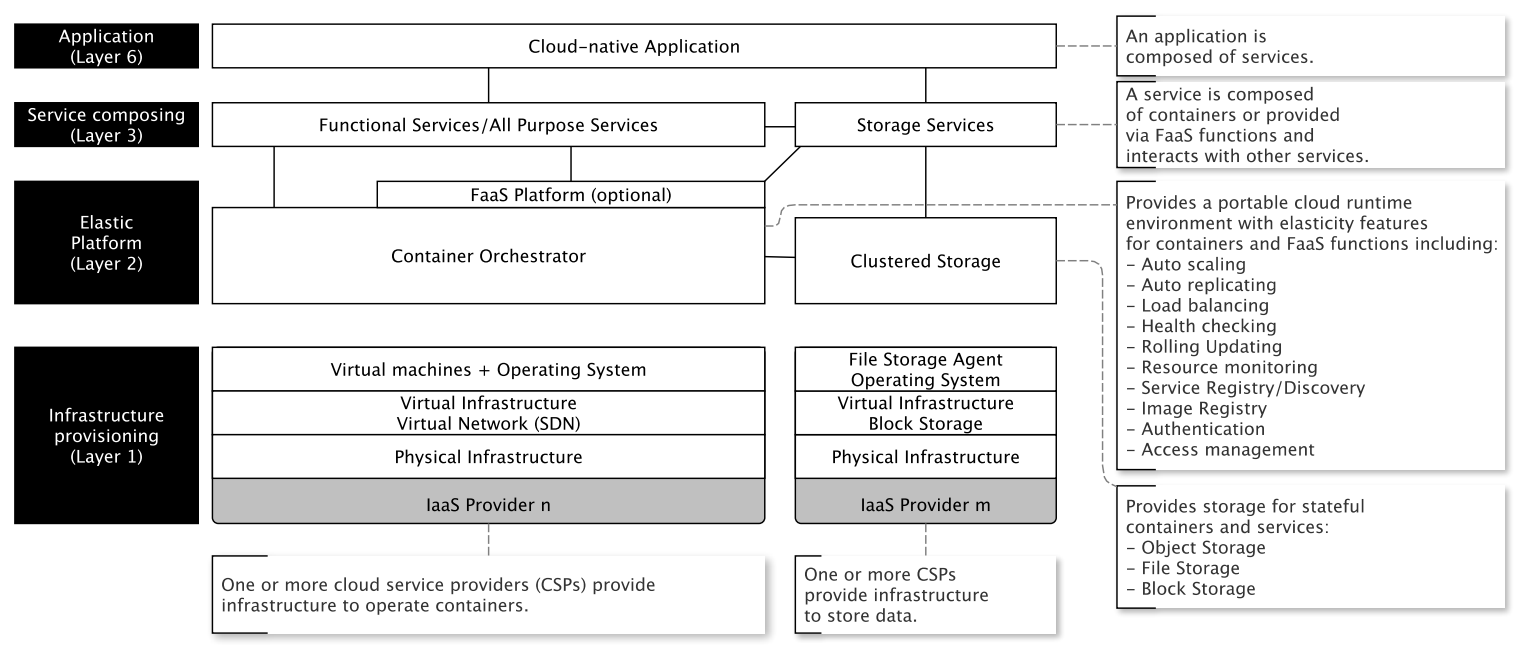

Figure 3. Cloud-native stack observable in a lot of cloud-native applications.

For more details, we refer to [3,5]. However, the remainder of this paper follows this model.

\section{Observable Long-Term Trends in Cloud Systems Engineering}

Cloud computing emerged some ten years ago. In the first adoption phase, existing IT-systems were merely transferred to cloud environments without changing the original design and architecture of these applications. Tiered applications were merely migrated from dedicated hardware to virtualized hardware in the cloud. Cloud system engineers implemented remarkable improvements in cloud 
platforms (PaaS) and infrastructures (IaaS) over the years and established several engineering trends currently observable.

All of these trends try to optimize specific quality factors like functional stability, performance efficiency, compatibility, usability, reliability, maintainability, portability, and security of cloud services to improve the overall quality of service (QoS). The most focused quality factors are functional stability, performance efficiency, and reliability (including availability) $[25,26]$. Therefore, these engineering trends listed in Table 2 seem somehow isolated. We want to review these trends from two different perspectives.

- In Section 5.1, we will investigate cloud application architectures from a resource utilization point of view over time.

- In Section 5.2, we will investigate cloud application architectures more from an evolutionary architecture point of view focusing mainly functional stability and reliability but also addressing compatibility, maintainability, and portability according to [26].

In both cases, we will see that the wish to make more efficient use of cloud resources had impacts on architectures and vice versa.

Table 2. Some observable software engineering trends coming along with cloud-native applications.

\begin{tabular}{ll}
\hline Trend & Rationale \\
\hline Microservices & $\begin{array}{l}\text { Microservices can be seen as a "pragmatic" interpretation of SOA. In addition to SOA, } \\
\text { microservice architectures intentionally focus and compose small and independently } \\
\text { replaceable horizontally scalable services that are "doing one thing well." [27-31] }\end{array}$ \\
\hline & $\begin{array}{l}\text { DevOps is a practice that emphasizes the collaboration of software developers and IT } \\
\text { operators. It aims to build, test, and release software more rapidly, frequently, and more } \\
\text { reliably using automated processes for software delivery [32,33]. DevOps foster the need } \\
\text { for independent replaceable and standardized deployment units and therefore pushes } \\
\text { microservice architectures and container technologies. }\end{array}$ \\
\hline Cloud Modeling & $\begin{array}{l}\text { Softwareization of infrastructure and network enables to automate the process of software } \\
\text { delivery and infrastructure changes more rapidly. Cloud modeling languages can express } \\
\text { applications and services and their elasticity behavior that shall be deployed to such } \\
\text { infrastructures or platforms. There is a good survey on this kind of new "programming } \\
\text { languages" [34]. }\end{array}$ \\
\hline $\begin{array}{l}\text { Standardized } \\
\text { Deployment }\end{array}$ & $\begin{array}{l}\text { Deployment units wrap a piece of software in a complete file system that contains } \\
\text { everything needed to run: code, runtime, system tools, system libraries. Thus, it is } \\
\text { guaranteed that the software will always run the same, regardless of its environment. } \\
\text { This deployment approach is often made using container technologies (OCI standard [19]) }\end{array}$ \\
& $\begin{array}{l}\text { Unikernels would work as well but are not yet in widespread use. A deployment unit } \\
\text { should be designed and interconnected according to a collection of cloud-focused patterns } \\
\text { like the twelve-factor app collection [35], the circuit breaker pattern [36] or cloud computing } \\
\text { patterns [37,38]. }\end{array}$ \\
\hline
\end{tabular}


Table 2. Cont.

\begin{tabular}{ll}
\hline Trend & Rationale \\
\hline Elastic Platforms & $\begin{array}{l}\text { Elastic platforms like Kubernetes [39], Mesos [47], or Swarm can be seen as a unifying } \\
\text { middleware of elastic infrastructures. Elastic platforms extend resource sharing and } \\
\text { increase the utilization of underlying compute, network and storage resources for custom } \\
\text { but standardized deployment units. }\end{array}$ \\
\hline the term serverless is used for an architectural style that is used for cloud application \\
architectures that deeply depend on external third-party-services (Backend-as-a-Service, \\
BaaS) and integrating them via small event-based triggered functions (Function-as-a, FaaS). \\
$\begin{array}{l}\text { FaaS extend resource sharing of elastic platforms by simply by applying time-sharing } \\
\text { concepts [40-42]. }\end{array}$ \\
\hline $\begin{array}{l}\text { State Isolation } \\
\text { Of course, stateful components cannot be avoided, but stateful components should be } \\
\text { reduced to a minimum and realized by intentional horizontal scalable storage systems } \\
\text { (often eventual consistent NoSQL databases) [37]. }\end{array}$ \\
\hline Rersioned REST & $\begin{array}{l}\text { REST-based APIs provide scalable and pragmatic communication, means relying mainly } \\
\text { on already existing internet infrastructure and well defined and widespread standards [43]. }\end{array}$ \\
\hline Loose coupling & $\begin{array}{l}\text { Service composition is done by events or by data [43]. Event coupling relies on messaging } \\
\text { solutions (e.g., AMQP standard). Data coupling often relies on scalable but (mostly) } \\
\text { eventual consistent storage solutions (which are often subsumed as NoSQL databases) [37]. }\end{array}$ \\
\hline
\end{tabular}

\subsection{A Review of the Resource Utilization Evolution and Its Impact on Cloud Technology Architectures}

Cloud infrastructures (IaaS) and platforms (PaaS) are built to be elastic. Elasticity is understood as the degree to which a system adapts to workload changes by provisioning and de-provisioning resources automatically. Without this, cloud computing is very often not reasonable from an economic point of view [1]. Over time, system engineers learned to understand this elasticity options of modern cloud environments better. Eventually, systems were designed for such elastic cloud infrastructures, which increased the utilization rates of underlying computing infrastructures via new deployment and design approaches like containers, microservices or serverless architectures. This design intention is often expressed using the term "cloud-native."

Figure 4 shows a noticeable trend over the last decade. Machine virtualization was introduced to consolidate plenty of bare metal machines to make more efficient utilization of physical resources. This machine virtualization forms the technological backbone of IaaS cloud computing. Virtual machines might be more lightweight than bare metal servers, but they are still heavy, especially regarding their image sizes. Due to being more fine-grained, containers improved the way of standardized deployments but also increased the utilization of virtual machines. Nevertheless, although containers can be scaled quickly, they are still always-on components. In addition, "recently," Function-as-a-Service (FaaS) approaches emerged and applied time sharing of containers on underlying container platforms. Using FaaS only, units are executed that have requests to be processed. Due to this time-shared execution of containers on the same hardware, FaaS enables even a scale-to-zero capability. This improved resource efficiency can be even measured monetarily [44]. Thus, over time, the technology stack to manage resources in the cloud got more complicated and harder to understand but followed one trend-to run more workload on the same amount of physical machines. 


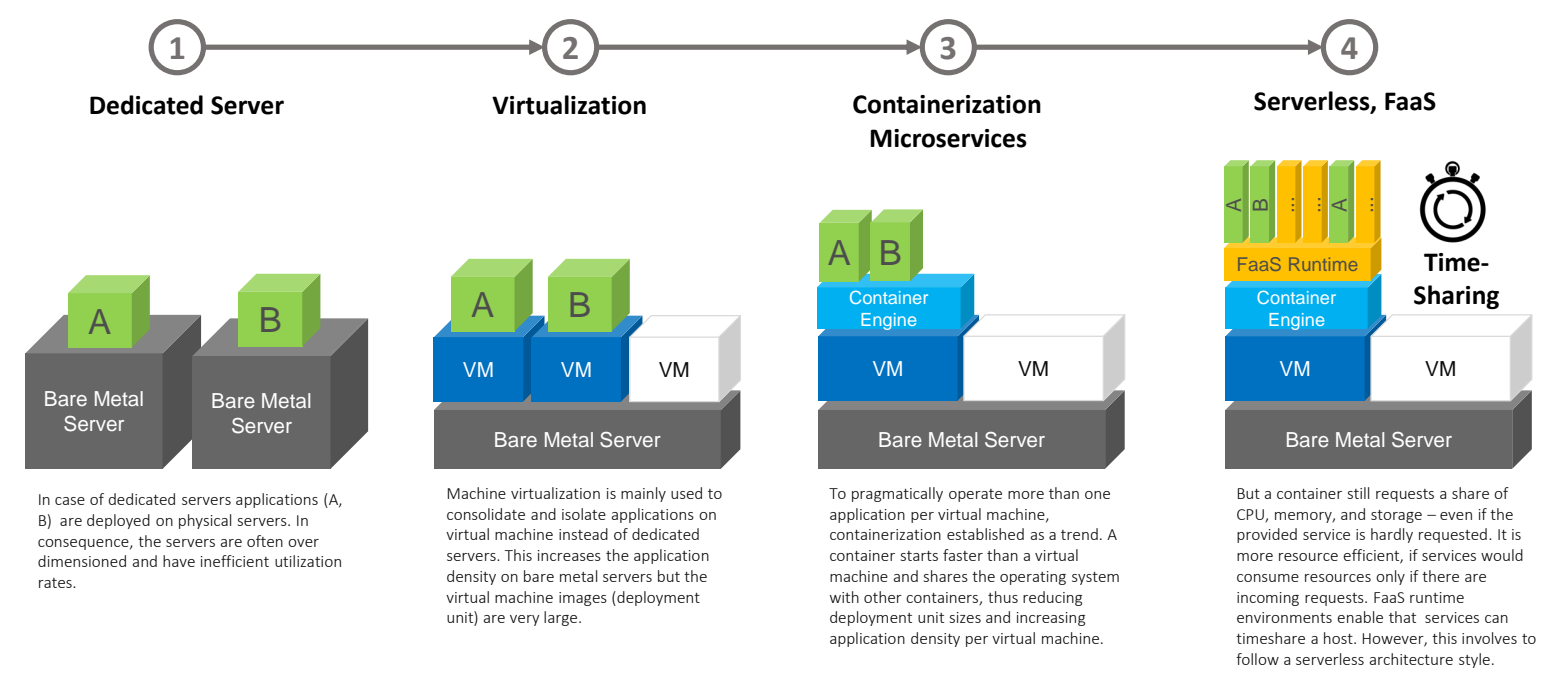

Figure 4. The cloud architectural evolution from a resource utilization point of view.

\subsubsection{Service-Oriented Deployment Monoliths}

An interesting paper the reader should dive into is [45]. Service-Oriented Computing (SOC) is a paradigm for distributed computing and e-business processing and has been introduced to manage the complexity of distributed systems and to integrate different software applications. A service offers functionalities to other services mainly via message passing. Services decouple their interfaces from their implementation. Workflow languages are used to orchestrate more complex actions of services (e.g., WS-BPEL). Corresponding architectures for such kind of applications are called Service-Oriented Architectures (SOA) consequently. Many business applications have been developed over the last decades following this architectural paradigm. In addition, due to its underlying service concepts, these applications can be deployed in cloud environments without any problems. Thus, they are cloud ready/friendly according to Table 1 . However, the main problem for cloud system engineers emerges from the problem that-although these kinds of applications are composed of distributed services-their deployment is not! These kinds of distributed applications are conceptually monolithic applications from a deployment point of view. Dragoni et al. define such monolithic software as:

"A monolithic software application is a software application composed of modules that are not independent of the application to which they belong. Since the modules of a monolith depend on said shared resources, they are not independently executable. This makes monoliths difficult to naturally distribute without the use of specific frameworks or ad hoc solutions [...]. In the context of cloud-based distributed systems, this represents a significant limitation, in particular, because previous solutions leave synchronization responsibilities to the developer [45]".

In other words, the complete distributed application must be deployed all at once in the case of updates or new service releases. This monolithic style even leads to situations where complete applications are simply packaged as one large virtual machine image. This fits perfectly to situations shown in Figure 4(1+2). However, depending on the application size, this normally involves noteworthy downtimes of the application for end users and limits the capability to scale the application in the case of increasing or decreasing workloads. While this might be acceptable for some services (e.g., some billing batch processes running somewhere in the night), it might be problematic for other kinds of services. What if messaging services (e.g., WhatsApp), large-scale social networks (e.g., Facebook), credit card instant payment services (e.g., Visa), traffic-considering navigational services (e.g., Google Maps), or ridesharing services (e.g., Uber) would go down for some hours just because of a new service release or a scaling operation? 
It is evident that especially cloud-native applications come along with such $24 \times 7$ requirements and the need to deploy, update, or scale single components independently from each other at runtime without any downtime. Therefore, SOA evolved into a so-called microservice architectural style. One might mention that microservices are mainly a more pragmatic version of SOA. Furthermore, microservices are intentionally designed to be independently deployable, updateable, and horizontally scalable. Thus, microservices have some architectural implications that will be investigated in Section 5.2.1. However, deployment units should be standardized and self-contained as well in this setting. We will have a look at that in the following Section 5.1.2.

\subsubsection{Standardized and Self-Contained Deployment Units}

While deployment monoliths are mainly using IaaS resources in the form of virtual machines that are deployed and updated less regularly, microservice architectures split up the monolith into independently deployable units that are deployed and terminated much more frequently. Furthermore, this deployment is done in a horizontally scalable way that is very often triggered by request stimuli. If many requests are hitting a service, more service instances are launched to distribute the requests across more instances. If the requests are decreasing, service instances are shut down to free resources (and save money). Thus, the inherent elasticity capabilities of microservice architectures are much more in focus compared with classical deployment monoliths and SOA approaches. One of the critical success factors for microservice architectures gaining so much attraction over the last years might be the fact that the deployment of service instances could be standardized as self-contained deployment units-so-called containers [46]. Containers make use of operating system virtualization instead of machine virtualization (see Figure 5) and are therefore much more lightweight. Containers enable making scaling much more pragmatic, and faster and, because containers are less resource consuming compared with virtual machines, the instance density on underlying IaaS hardware could be improved.

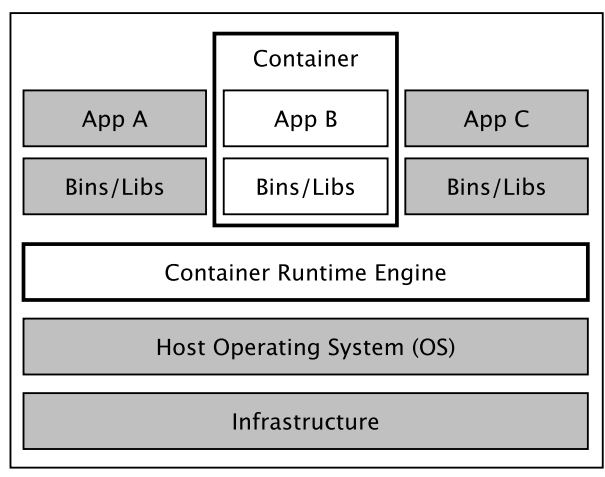

Containers:

Containers are an abstraction at the application layer that packages code and dependencies together. Multiple containers can run on the same machine and share the OS kernel with other containers, each running as isolated processes in user space. Containers take up less space than VMs (typically tens of MBs) and start seconds to milliseconds.

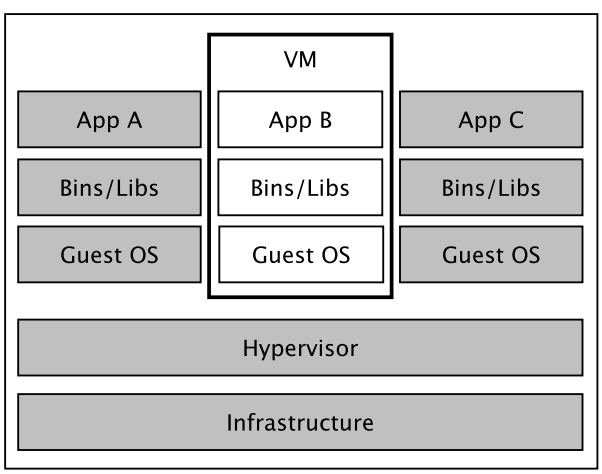

Virtual Machines:

Virtual machines (VM) are an abstraction of physical hardware turning one server into many servers. The hypervisor allows multiple VMs to run on a single machine. Each VM includes a full copy of an operating system, one or more apps, necessary binaries and libraries - taking often up tens of GBs. VMs take normally minutes to boot.

Figure 5. Comparing containers and virtual machines (adapted from the Docker website).

However, even in microservice architectures, the service concept is an always-on concept. Thus, at least one service instance (container) must be active and running for each microservice at all times. Microservice architectures make use of plenty of such small services. To have a lot of small services is the dominant design philosophy of the microservice architectural approach. Thus, even container technologies do not overcome the need for always-on components. In addition, always-on components are some of the most expensive and therefore avoidable cloud workloads according to 
Weinmann [1]. Thus, the question arises of whether it is possible to execute service instances only in the case of actual requests. Moreover, the answer leads to Function-as-a-Service concepts and corresponding platforms that will be discussed in Section 5.1.3.

\subsubsection{Function-as-a-Service}

Microservice architectures propose a solution to efficiently scale computing resources that are hardly realizable with monolithic architectures [45]. The allocated infrastructure can be better tailored to the microservices' needs due to the independent scaling of each one of them via standardized deployment units addressed in Section 5.1.2. However, microservice architectures face additional efforts like deploying every single microservice and scaling and operating them in cloud infrastructures. To address these concerns, container orchestrating platforms like Kubernetes [39], or Mesos/Marathon [47] emerged. However, this shifts the problem mainly to the operation of these platforms and these platforms are still always-on components. Thus, so-called Serverless architectures and Function-as-a-Service platforms have emerged in the cloud service ecosystem. The AWS lambda service might be the most prominent one, but there exist more like Google Cloud Functions, Azure Functions, OpenWhisk, Spring Cloud Functions to name just a few. However, all (commercial platforms) follow the same principle to provide very small and fine-grained services (just exposing one stateless function) that are billed on a runtime-consuming model (millisecond dimension). The problem with the term Serverless is that it occurs in two different notions.

1. "Serverless was first used to describe applications that significantly or fully incorporate third-party, cloud-hosted applications and services, to manage server-side logic and state. These are typically "rich client" applications - think single-page web apps, or mobile apps-that use the vast ecosystem of cloud-accessible databases, authentication services, and so on. These types of services can be described as "Backend as a Service (BaaS) [40]".

2. "Serverless can also mean applications where server-side logic is still written by the application developer, but, unlike traditional architectures, it is run in stateless compute containers that are event-triggered, ephemeral (may only last for one invocation), and fully managed by a third party. One way to think of this is "Functions as a Service" or "FaaS." AWS Lambda is one of the most popular implementations of a Functions-as-a-Service platform at present, but there are many others, too [40]".

In this section, we use the term Serverless computing in the notion of FaaS, and we will mainly investigate the impact on resource utilization. The upcoming Section 5.2.2 will investigate Serverless more in architectural terms. FaaS was specifically designed for event-driven applications that require carrying out lightweight processing in response to an event [48]. FaaS is more fine-grained than microservices and facilitates the creation of functions. Therefore, these fine-grained functions are sometimes called nanoservices. These functions can be easily deployed and automatically scaled, and provide the potential to reduce infrastructure and operation costs. Others like the deployment unit approaches of Section 5.1.2 — that are still always-on software components-functions are only processed if there are active requests. Thus, FaaS can be much more cost efficient than just containerized deployment approaches. According to a cost comparison of monolithic, microservice and FaaS architectures case study by Villamizar et al. cost reductions up to $75 \%$ are possible [44]. On the other hand, there are still open problems like the Serverless trilemma identified by Baldini et al. The Serverless trilemma "captures the inherent tension between economics, performance, and synchronous composition" [42] of serverless functions. One obvious problem stressed by Baldini et al. is the "double spending problem" shown in Figure 6. This problem occurs when a serverless function $f$ is calling another serverless function $g$ synchronously. In this case, the consumer is billed for the execution of $f$ and $g$-although only $g$ is consuming resources because $f$ is waiting for the result of $g$. To avoid this double spending problem, a lot of serverless applications delegate the composition of fine-grained serverless functions into higher order functionality to client applications and edge devices 
outside the scope of FaaS platforms. This composition problem leads to new-more distributed and decentralized-forms of cloud-native architectures investigated in Section 5.2.2.

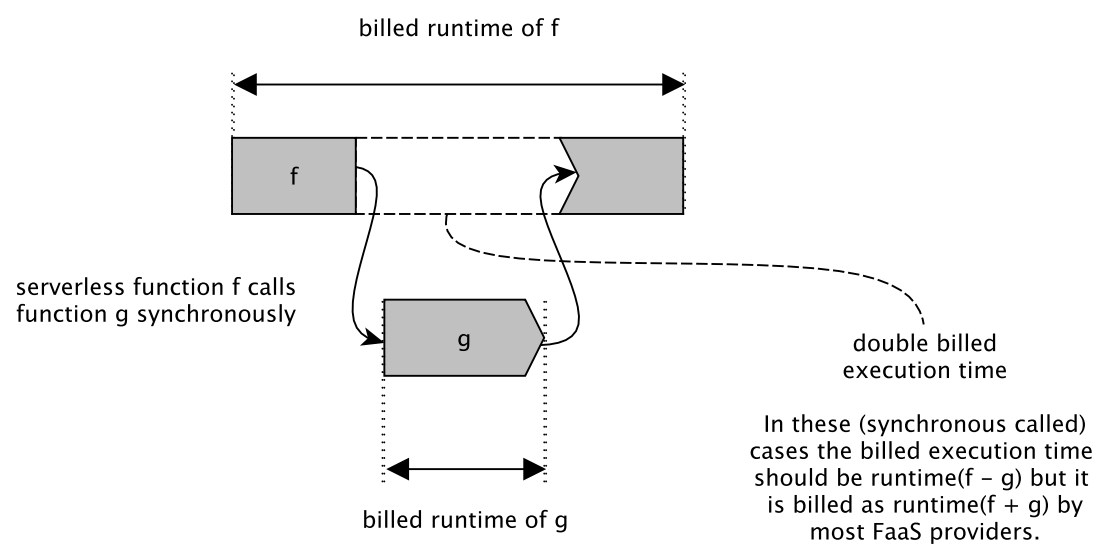

Figure 6. The double spending problem resulting from the Serverless trilemma [42].

\subsection{A Review of the Architectural Evolution}

The reader has seen in Section 5.1 that Cloud-native applications strived for a better resource utilization mainly by applying more fine-grained deployment units in the shape of lightweight containers (instead of virtual machines) or shape of functions in the case of FaaS approaches. Moreover, these improvements of resource utilization rates had an impact on how architectures of cloud applications evolved. Two major architectural trends of Cloud application architectures established in the last decade. We will investigate Microservice architectures in Section 5.2.1 and Serverless architectures in Section 5.2.2.

\subsubsection{Microservice Architectures}

Microservices form "an approach to software and systems architecture that builds on the well-established concept of modularization but emphasize technical boundaries. Each module - each microservice-is implemented and operated as a small yet independent system, offering access to its internal logic and data through a well-defined network interface. This architectural style increases software agility because each microservice becomes an independent unit of development, deployment, operations, versioning, and scaling [31]". According to [30,31], often mentioned benefits of microservice architectures are faster delivery, improved scalability, and greater autonomy. Different services in a microservice architecture can be scaled independently from each other according to their specific requirements and actual request stimuli. Furthermore, each service can be developed and operated by different teams. Thus, microservices do not only have a technological but also an organizational impact. These teams can make localized decisions per service regarding programming languages, libraries, frameworks, and more. Thus, best-of-breed breaches are possible within each area of responsibility on the one hand - on the other hand, this might increase the technological heterogeneity naturally across the complete system, and corresponding long-term effects regarding maintainability of such systems might be not even observed so far [4].

Alongside microservice architectures, we observed several other accompanying trends. We already investigated containerization as such a trend in Section 5.1.2. First generation microservices formed of individual services that were packed using container technologies (see Figure 7). These services were then deployed and managed at runtime using container orchestration tools, like Mesos. Each service was responsible for keeping track of other services, and invoking them by specific communication protocols. Failure-handling was implemented directly in the services' source code. With an increase of services per application, the reliable and fault-tolerant location and invocation of appropriate service instances became a problem itself. If new services were implemented 
using different programming languages, but made reusing existing discovery and failure-handling code become increasingly difficult. Thus, freedom of choice and "polyglot programming" are an often mentioned benefit of microservices but has its drawbacks that need to be managed.

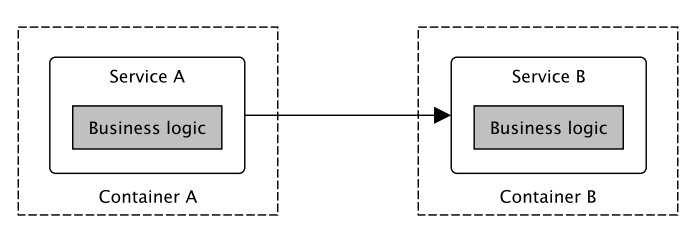

(a) 1st generation microservice

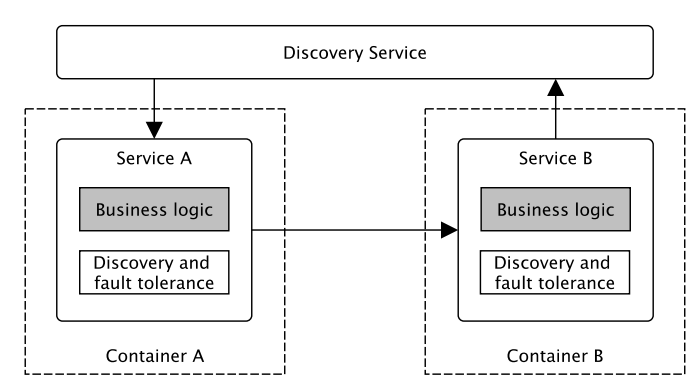

(b) 2nd generation microservice

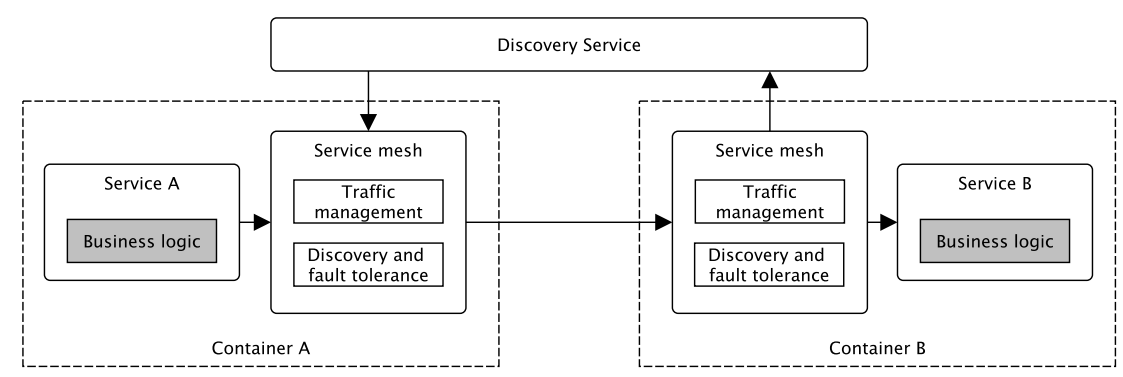

(c) 3rd generation microservice

Figure 7. Microservice architecture evolution—adapted from [31].

Therefore, second generation microservice architectures (see Figure 7) made use of discovery services and reusable fault-tolerant communication libraries. Common discovery services (like Consul) were used to register provided functionalities. During service invocation, all protocol-specific and failure-handling features were delegated to an appropriate communication library, such as Finagle. This simplified service implementation and reuse of boilerplate communication code across services.

The third generation (see Figure 7) introduced service proxies as transparent service intermediates with the intent to improve software reusability. So-called sidecars encapsulate reusable service discovery and communication features as self-contained services that can be accessed via existing fault-tolerant communication libraries provided by almost every programming language nowadays. Because of its network intermediary conception, sidecars are more than suited for monitoring the behavior of all service interactions in a microservice application. This intermediary is precisely the idea behind service mesh technologies such as Linkerd. These tools extend the notion of self-contained sidecars to provide a more integrated service communication solution. Using service meshes, operators have much more fine-grained control over the service-to-service communication including service discovery, load balancing, fault tolerance, message routing, and even security. Thus, besides the pure architectural point of view, the following tools, frameworks, services, and platforms (see Table 3) form our current understanding of the term microservice:

- Service discovery technologies let services communicate with each other without explicitly referring to their network locations.

- Container orchestration technologies automate container allocation and management tasks and abstracting away the underlying physical or virtual infrastructure from service developers. This is the reason that we see this technology as an essential part of any cloud-native application stack (see Figure 3). 
- Monitoring technologies that are often based on time-series databases to enable runtime monitoring and analysis of the behavior of microservice resources at different levels of detail.

- Latency and fault-tolerant communication libraries let services communicate more efficiently and reliably in permanently changing system configurations with plenty of service instances permanently joining and leaving the system according to changing request stimuli.

- Continuous-delivery technologies integrate solutions often into third-party services that automate many of the DevOps practices typically used in a web-scale microservice production environment [32].

- Service proxy technologies encapsulate mainly communication-related features such as service discovery and fault-tolerant communication and expose them over HTTP.

- Finally, latest service mesh technologies built on sidecar technologies to provide a fully integrated service-to-service communication monitoring and management environment.

Table 3. Some observable microservice engineering ecosystem components (adapted from [31]).

\begin{tabular}{|c|c|}
\hline $\begin{array}{l}\text { Ecosystem } \\
\text { Component }\end{array}$ & Example Tools, Frameworks, Services and Platforms (Last Access 07/11/2018) \\
\hline $\begin{array}{l}\text { Service } \\
\text { discovery }\end{array}$ & $\begin{array}{l}\text { Zookeeper (https: / / zookeeper.apache.org), Eureka (https:/ / github.com/Netflix/eureka), } \\
\text { Consul (https:/ / www.consul.io), etcd (https:/ / github.com/coreos/etcd, Synapse (https: } \\
\text { //github.com/airbnb/synapse) }\end{array}$ \\
\hline $\begin{array}{l}\text { Container } \\
\text { orchestration }\end{array}$ & $\begin{array}{l}\text { Kubernetes (https:/ / kubernetes.io, [39]), Mesos (http:/ / mesos.apache.org, [47], Swarm } \\
\text { (https:/ / docs.docker.com/engine/swarm), Nomad (https:/ / www.nomadproject.io) }\end{array}$ \\
\hline Monitoring & $\begin{array}{l}\text { Graphite (https:/ / graphiteapp.org), InfluxDB (https://github.com/influxdata/influxdb), } \\
\text { Sensu (https:// sensuapp.org), cAdvisor (https://github.com/google/cadvisor), } \\
\text { Prometheus (https:// prometheus.io), Elastic Stack (https://elastic.co/elk-stack) }\end{array}$ \\
\hline $\begin{array}{l}\text { Fault tolerant } \\
\text { communication }\end{array}$ & $\begin{array}{l}\text { Finagle (https:/ / twitter.github.io/finagle), Hystrix (https:/ / github.com/Netflix/Hystrix), } \\
\text { Proxygen (https:/ /github.com/facebook/proxygen), Resilience4j (https:/ /github.com/ } \\
\text { resilience4j) }\end{array}$ \\
\hline $\begin{array}{l}\text { Continuous } \\
\text { delivery services }\end{array}$ & $\begin{array}{l}\text { Ansible (https://ansible.com), Circle CI (https://circleci.com/), Codeship (https:// } \\
\text { codeship.com/), Drone (https://drone.io), Spinnaker (https://spinnaker.io), Travis CI } \\
\text { (https://travis-ci.org/) }\end{array}$ \\
\hline Service proxy & Prana (https:/ / github.com/Netflix/Prana), Envoy (https://www.envoyproxy.io) \\
\hline Service meshs & Linkerd (https: / / linkerd.io), Istio (https://istio.io) \\
\hline
\end{tabular}

Table 3 shows that a complex tool-chain evolved to handle the continuous operation of microservice-based cloud applications.

\subsubsection{Serverless Architectures}

Serverless computing is a cloud computing execution model in which the allocation of machine resources is dynamically managed and intentionally out of control of the service customer. The ability to scale to zero instances is one of the critical differentiators of serverless platforms compared with container focused PaaS, or virtual machine focused IaaS services. Scale-to-zero enables avoiding billed always-on components and therefore excludes the most expensive cloud usage pattern according to [1]. That might be one reason why the term "serverless" is getting more and more common since 2014 [31]. However, what is "serverless" exactly? Servers must still exist somewhere.

So-called serverless architectures replace server administration and operation mainly by using Function-as-a-Service (FaaS) concepts [40] and integrating third-party backend services. Figure 4 showed the evolution of how resource utilization has been optimized over the last ten years ending in the latest trend to make use of FaaS platforms. FaaS platforms apply time-sharing principles and increase the utilization factor of computing infrastructures, and thus avoid expensive always-on components. As already mentioned, at least one study showed that, due to this time-sharing, serverless 
architectures can reduce costs by 70\% [44]. A serverless platform is merely an event processing system (see Figure 8). According to [42], serverless platforms take an event (sent over HTTP or received from a further event source in the cloud). Then, these platforms determine which functions are registered to process the event, find an existing instance of the function (or create a new one), send the event to the function instance, wait for a response, gather execution logs, make the response available to the user, and stop the function when it is no longer needed. Besides API composition and aggregation to reduce API calls [42], event-based applications are especially very much suited for this approach [49].

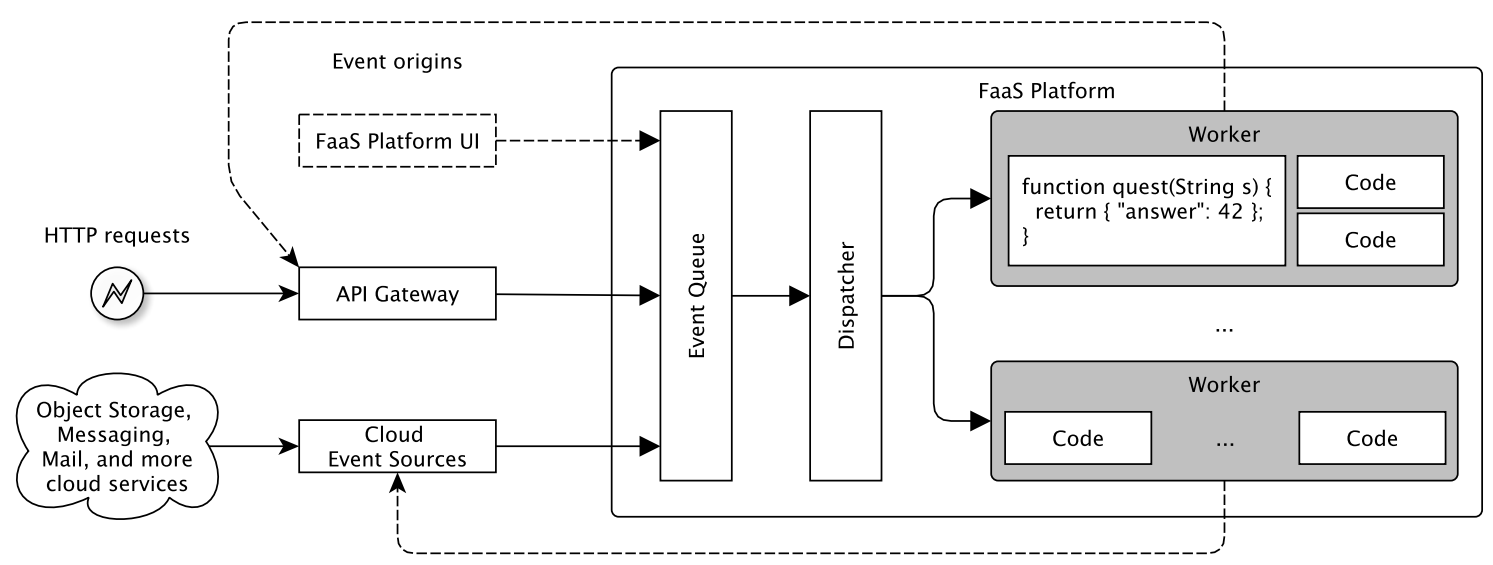

Figure 8. Blueprint of a serverless platform architecture (adapted from [42]).

Serverless platform provision models can be grouped into the following categories:

- Public (commercial) serverless services of public cloud service providers provide computational runtime-environments, also known as a function as a service (FaaS) platforms. Some well-known type representatives include AWS Lambda, Google Cloud Functions, or Microsoft Azure Functions. All of the mentioned commercial serverless computing models are prone to create vendor lock-in (to some degree).

- Open (source) serverless platforms like Apache's OpenWhisk or OpenLambda might be an alternative with the downside that these platforms need infrastructure.

- Provider agnostic serverless frameworks provide a provider and platform agnostic way to define and deploy serverless code on various serverless platforms or commercial serverless services. Thus, these frameworks are an option to avoid (or reduce) vendor lock-in without the necessity to operate an own infrastructure.

Thus, on the one hand, serverless computing provides some inherent benefits like resource and cost efficiency, operation simplicity, and a possible increase of development speed and better time-to-market [40]. However, serverless computing also comes along with some noteworthy drawbacks, like runtime constraints, state constraints and still unsatisfactorily solved function composition problems like the double spending problem (see Figure 6). Furthermore, resulting serverless architectures have security implications. They increase attack surfaces and shift parts of the application logic (service composing) to the client-side (which is not under complete control of the service provider). Furthermore, FaaS increases vendor lock-in problems, client complexity, as well as integration and testing complexity. Table 4 summarizes some of the most mentioned benefits but also drawbacks of FaaS from practitioner reportings [40]. 
Table 4. Serverless architecture benefits and drawbacks (mainly compiled from [40]).

\begin{tabular}{|c|c|}
\hline Benefits & Drawbacks \\
\hline $\begin{array}{l}\text { RESOURCE EFFIENCY (service side) } \\
\text { - auto-scaling based on event stimulus } \\
\text { - reduced operational costs } \\
\text { - scale to zero capability (no always-on) }\end{array}$ & $\begin{array}{l}\text { - maximum function runtime is limited } \\
\text { - startup latencies of functions must be considered } \\
\text { - function runtime variations } \\
\text { - functions can not preserve a state across function calls } \\
\text { - external state (cache, key/value stores, etc.) can } \\
\text { compensate this but is a magnitude slower } \\
\text { - double spending problems (FaaS functions call other } \\
\text { FaaS functions) }\end{array}$ \\
\hline $\begin{array}{l}\text { OPERATION (service side) } \\
\text { - simplified deployment } \\
\text { - simplified operation (see auto-scaling) }\end{array}$ & $\begin{array}{l}\text { - increased attack surfaces } \\
\text { - each endpoint introduces possible vulnerabilities } \\
\text { - missing protective barrier of a monolithic server } \\
\text { application } \\
\text { - parts of the application logic are shifted to the client-side } \\
\text { (that is not under control of the service provider) } \\
\text { - increased vendor lock-in (currently no FaaS standards } \\
\text { for API gateways and FaaS runtime environments) }\end{array}$ \\
\hline $\begin{array}{l}\text { DEVELOPMENT SPEED (service side) } \\
\text { - development speed } \\
\text { - simplified unit testing of stateless FaaS functions } \\
\text { - better time to market }\end{array}$ & $\begin{array}{l}\text { - increased client complexity } \\
\text { - application logic is shifted to the client-side } \\
\text { - code replication on client side across client platforms } \\
\text { - control of application workflow on client side to avoid } \\
\text { double-sending problems of FaaS computing } \\
\text { - increased integration testing complexity } \\
\text { - missing integration test tool-suites }\end{array}$ \\
\hline
\end{tabular}

Furthermore, Figure 9 shows that serverless architectures (and microservice architectures as well) require a cloud application architecture redesign, compared to traditional e-commerce applications. Much more than microservice architectures, serverless architectures integrate third-party backend services like authentication or database services intentionally. Functions on FaaS platforms provide only very service specific, security relevant, or computing intensive functionality. In fact, all functionality that would have been provided classically on a central application server is now provided as a lot of isolated micro- or even nanoservices. The integration of all these isolated services as meaningful end-user functionality is delegated to end devices (very often in the shape of native mobile applications or progressive web applications). In summary, we can see the following observable engineering decisions in serverless architectures:

- Former cross-sectional but service-internal (or via a microservice provided) logic like authentication or storage is sourced to external third party services.

- Even nano- and microservice composition is shifted to end-user clients or edge devices. This means that even service orchestration is not done anymore by the service provider itself but by the service consumer via provided applications. This end-user orchestration has two interesting effects: (1) the service consumer now provides resources needed for service orchestration; (2) because the service composition is done outside the scope of the FaaS platform, still unsolved FaaS function composition problems (like the double spending problem) are avoided.

- Such client or edge devices are interfacing third party services directly.

- Endpoints of very service specific functionality is provided via API gateways. Thus, HTTP- and REST-based/REST-like communication protocols are generally preferred.

- Only very domain or service specific functions are provided on FaaS platforms. Mainly, when this functionality is security relevant and should be executed in a controlled runtime environment by the service provider, or the functionality is too processing or data-intensive to be executed on 
consumer clients or edge devices, or the functionality is so domain-, problem-, or service-specific that simply no external third-party service exists.
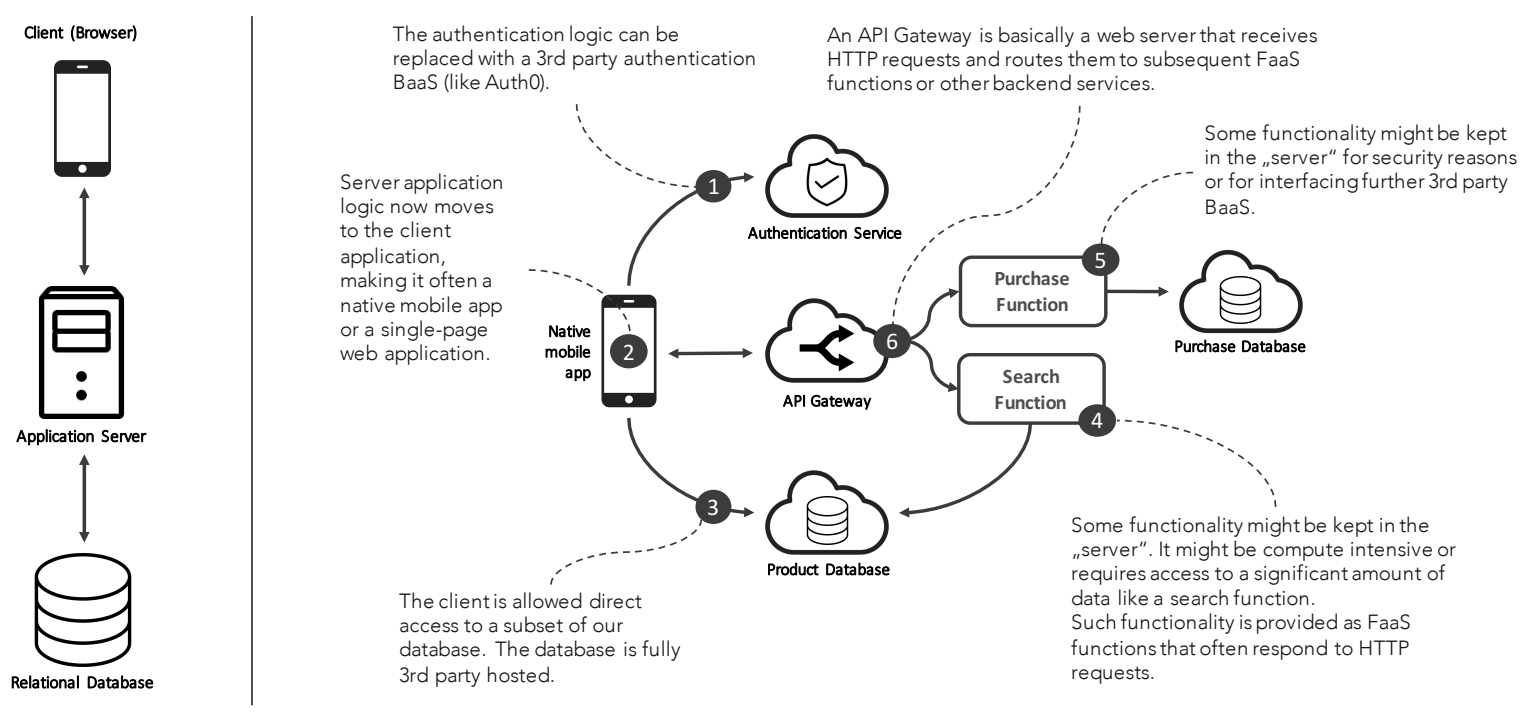

Figure 9. Serverless architectures result in a different and less centralized composition of application components and backend services compared with classical tiered application architectures.

Finally, the reader might observe the trend in serverless architectures that this kind of architecture is more decentralized and distributed, makes more intentional use of independently provided services, and is therefore much more intangible (more cloudy) compared with microservice architectures.

\section{The Road Ahead}

So far, we have identified and investigated two major trends. First, cloud computing and its related application architecture evolution can be seen as a steady process to optimize resource utilization in cloud computing. This was visualized in Figure 4 and discussed in Section 5.1. Second, in Section 5.2, it was emphasized that this resource utilization improvement results over time in an architectural evolution of how cloud applications are being built and deployed. We observed a shift from monolithic SOA, via independently deployable microservices towards so-called serverless architectures that are more decentralized and distributed, and make more intentional use of independently provided services.

The question is whether and how are these trends continuing? To forecast the future is challenging, but having current trends and the assumption that these trends will go on to some degree make it a bit easier. This forecast is done in Section 6.1 for the optimization of resource utilization trend, and Section 6.2 will take a look at how cloud application architectures may evolve in the future by merely extrapolating the existing SOA-microservice-serverless path.

\subsection{Unikernels—The Overlooked Deployment Unit?}

Operating system virtualization based container technologies have massively influenced the resource utilization optimization trend. However, containers are not about virtualization from a cloud application deployment point of view. They are about a standardized and self-contained way to define deployment units. However, are containers the only solution and the most resource efficient solution already existing? The answer is no, and roads ahead might follow directions with the same intent to define standardized and self-contained deployment units but with better resource utilization.

One option would be unikernels. A unikernel is a specialized, single address space machine image constructed via library operating systems. The first such systems were Exokernel (MIT Parallel and Distributed Operating Systems group) and Nemesis (the University of Cambridge, University of 
Glasgow, Swedish Institute of Computer Science and Citrix Systems) in the late 1990s. The basic idea is that a developer selects a minimal set of libraries which correspond to the OS constructs required for their application to run. These libraries are then compiled with the application and configuration code to build sealed, fixed-purpose images (unikernels) which run directly on a hypervisor or hardware without an OS. Thus, unikernels are self-contained deployment units like containers we investigated in Section 5.1.2 with the advantage to avoid a container overhead, a container runtime engine, and a host operating system (see Figure 5). Thus, interesting aspects to investigate on the road ahead would be:

- Because unikernels make operating systems and container runtime engines obsolete, this could further increase resource utilization rates.

- FaaS platforms workers are normally container based. However, unikernels are a deployment option as well. Interesting research and engineering directions would be how to combine unikernels with FaaS platforms to apply the same time-sharing principles.

However, although there is research following the long-term trend to improve resource utilization [50,51], most cloud computing-related unikernel research [52-55] mainly investigates unikernels as a security option to reduce attack surfaces (which are increased by serverless and microservice architectures as we have seen in Section 5.2). However, the resource optimization effect of unikernels might be still not aware to cloud engineers. Other than container technology, unikernel technology is not hyped.

\subsection{Overcoming Conceptual Centralized Approaches}

This section investigates some long-term trends in cloud and service computing research through the support of quantitative trend analysis. Scopus has been used to count the number of published papers dealing with some relevant terms over the years. We searched for the following terms in titles, abstracts, or keywords limited to the computer science domain:

- Cloud computing - to collect the amount of cloud computing-related research in general.

- $S O A$ - to collect the service computing related research, which is still a major influencing concept in cloud computing.

- Microservices - to collect microservice related research (which is more modern and pragmatic interpretation of SOA and very popular in cloud computing).

- Serverless - to collect serverless architecture related research (which is the latest observable architecture trend in cloud computing).

- Peer-to-peer (P2P) — to collect P2P related research (because recently more decentralizing concepts are entering cloud computing).

- Blockchain-to collect blockchain related research (which is the latest observable P2P related research trend/hype).

The presented architectural evolution can be seen as the perpetual fight of centralism and decentralism. Centralized architectures are known since decades. This kind of architectures makes system engineering easier. Centralized architectures have fewer problems with data synchronization and data redundancy. They are easier to handle from a conceptual point of view. The client-server architecture is still one of the most basic but dominant centralized architectural styles.

However, at various point in times, centralized approaches are challenged by more decentralized approaches. Take the mainframe versus personal computer as one example dating back to the 1980s. Currently, such decentralizing trends often correlate with terms like mobile cloud computing [56], the Internet of Things (IoT) and edge computing. Edge computing is a method for cloud computing systems to hand over control of services from some central nodes (the "core") to the "edge" of the Internet which makes contact with the physical world. Data comes in from the physical world via various sensors, and actions are taken to change physical state via various forms of actuators. By performing analytics and knowledge generation at the edge, communications bandwidth between 
systems and central data processing can be reduced. Edge computing takes advantage of proximity to the physical items of interest also exploiting relationships those items may have to each other but needs more decentralized processing approaches. Thus, the question arises how conceptually centralized service-oriented solutions can be adapted for such more decentralized problems $[57,58]$.

Figure 10 shows the number of papers per year for research that is dealing with cloud computing in general and relates it with serverless architectures, P2P based related research (including blockchains as a latest significant P2P trend). We see a rise in interest in research about peer-to-peer (that means decentralized) approaches starting in 2000 that reached its peak in 2010. What is interesting to us is that peer-to-peer based research decreased with the beginning starting increase of cloud computing related research in 2008. Thus, cloud computing (mainly a concept to provide services in a conceptually centralized manner) decreased the interest in peer-to-peer related research. P2P computing is a distributed application architecture that partitions tasks or workloads between peers. Peers are equally privileged and equipotent participants in the application. Peers make a portion of their resources, such as processing power, disk storage or network bandwidth, directly available to other network participants, without the need for central coordination by servers or stable hosts. Thus, peers are both suppliers and consumers of resources, in contrast to the cloud computing consumer-service model. However, leveraging P2P technologies for service provisioning has been identified of particular interest for research, and one of the challenges is how to enable the service providers to adapt themselves in response to changing service demand [59].

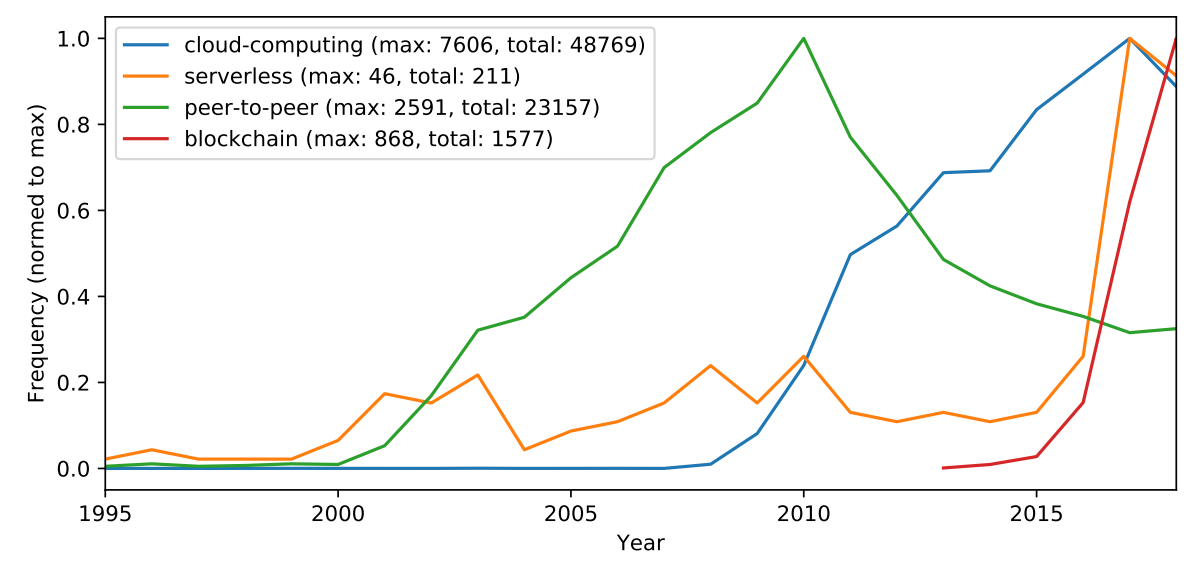

Figure 10. Trends of papers dealing with the terms cloud-computing, serverless, P2P, and blockchain (as the latest P2P based trend). Retrieved from Scopus (limited to computer science), 2018 extrapolated.

One astonishing curve in Figure 10 is the research interest in serverless solutions. Although on a substantially lower absolute level, a constant research interest in serverless solutions can be observed since 1995. To have "serverless" solutions seems to be a long-standing dream in computer science. The reader should be aware that the notion of serverless changed over time. Serverless has been used until 2000 very often in file storage research contexts. With the rise of P2P based solutions, the term serverless has been mainly used alongside P2P based approaches. In addition, since 2015, much momentum has been given alongside cloud-native application architectures (see Figure 11). Thus, nowadays, it is mainly used in the notion described in Sections 5.1.3 and 5.2.2. 


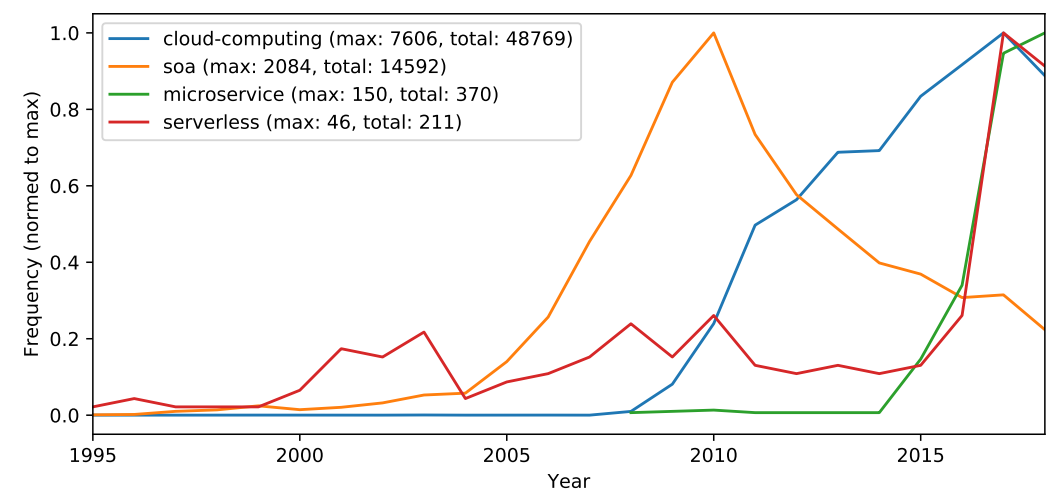

Figure 11. Trends of papers dealing with cloud-computing, SOA, microservices and serverless. Retrieved from Scopus (limited to computer science), 2018 extrapolated.

Figure 11 shows some further interesting correlation. With the rise of cloud computing in 2008, there is a steady decline in SOA related research. Thus, to deploy monolithic SOA applications in the cloud was not seen useful from the very beginning of cloud computing. However, it took almost five years in research to investigate further and more cloud suited application architectures (microservice and serverless architectures).

If we look at Figures 10 and 11, we see a decline of classical architecture approaches like SOA and a rising interest in new architecture styles like microservice and serverless architectures. Again, especially serverless architectures come along with some decentralizing philosophy that is observable in P2P based research as well. The author does not think that those cloud application architectures will strive for the same level of decentralizing and distribution like peer-to-peer based approaches. However, a more distributed service-to-service trend is observable in cloud application architecture research [60]. Thus, the cloud computing trend started a decline in SOA (see Figure 11) and P2P (see Figure 10). However, if we compare SOA and P2P (including blockchain related research), we see an increasing interest in decentralized solutions again (see Figure 12).

If we are taking all this together to forecast the road ahead, we could assume that new architecture styles like microservices and serverless architectures will dominate service computing. Moreover, SOA seems to die. However, we see a resurgence of interest in decentralized approaches known from P2P related research. Therefore, the author assumes that especially serverless architectures will more and more evolve into cloud application architectures that follow distributed service-to-service principles (much more in the notion of peer-to-peer).

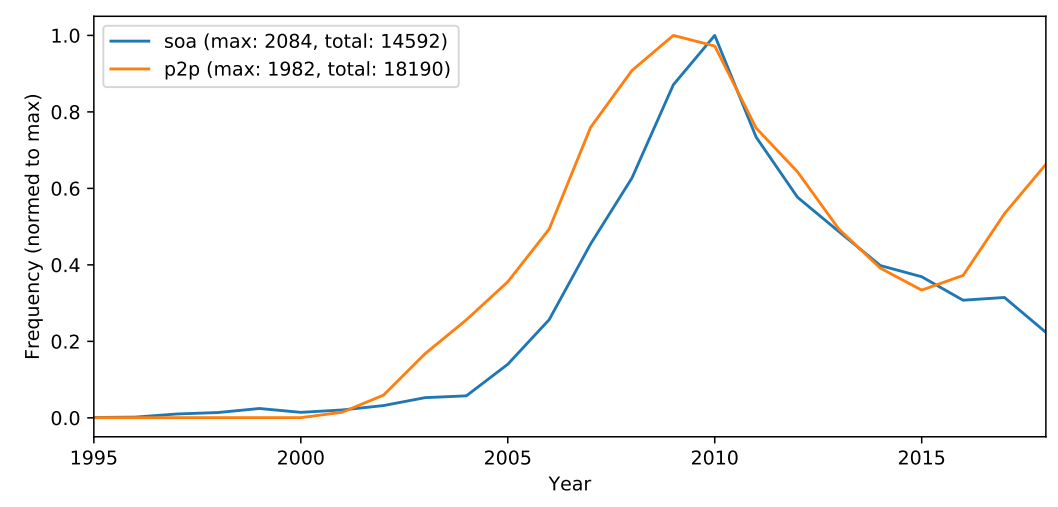

Figure 12. Trends of papers dealing with SOA, and P2P (including blockchain). Retrieved from Scopus (limited to computer science), 2018 extrapolated. 


\section{Related Work}

As far as the author knows, no survey focused intentionally observable trends in cloud applications' architectures over the last decade from a "big picture" architectural evolution point of view. This paper grouped that evolution mainly into the following point of views:

- Resource utilization optimization approaches like containerization and FaaS approaches have been investigated in Section 5.1.

- The architectural evolution of cloud applications that is dominated by microservices and evolving into serverless architectures. Both architectural styles have been investigated in Section 5.2.

For all of these four specific aspects (containerization, FaaS, microservices, serverless architectures), there exist surveys that should be considered by the reader. The studies and surveys [46,61-63] deal mainly with containerization and its accompanying resource efficiency. Although FaaS is quite young and could be only little reflected in research so far, there exist first survey papers [42,64-67] dealing with FaaS approaches deriving some open research questions regarding tool support, performance, patterns for serverless solutions, enterprise suitability and whether serverless architectures will extend beyond traditional cloud platforms and architectures.

Service composition provides value-adding and higher-order services by composing basic services that can be even pervasively provided by various organizations $[68,69]$. Furthermore, service computing is quite established, and there are several surveys on SOA related aspects [70-74]. However, more recent studies focus mainly on microservices. Refs. [29,31,45] focus especially on the architectural point of view and the relationship between SOA and microservices. All of these papers are great to understand the current microservice "hype" better. It is highly recommended to study these papers. However, these papers are somehow bound to microservices and do not take the "big picture" of general cloud application architecture evolution into account. Ref. [31] provides a great overview of microservices and even serverless architectures, but serverless architectures are subsumed as a part of microservices to some degree. The author is not quite sure whether serverless architectures do not introduce fundamental new aspects into cloud application architectures that evolve from the "scale-to-zero" capability on the one hand and the unsolved function composition aspects (like the double spending problem) on the other hand. Resulting serverless architectures push former conceptually centralized service composing logic to end user and edge devices out of direct control of the service provider.

\section{Conclusions}

Two major trends in cloud application architecture have been identified and investigated. First, cloud computing and its related application architecture evolution can be seen as a steady process to optimize resource utilization in cloud computing. Unikernels-a technology from late 1990s-might be one option for future improvements. Like containers, they are self-contained but avoid a container overhead, a container runtime engine, and even a host operating system. However, astonishing little research is conducted in that field. Second, each resource utilization improvement resulted in an architectural evolution of how cloud applications are being built and deployed. We observed a shift from monolithic SOA (machine virtualization), via independently deployable microservices (container) towards so-called serverless architectures (FaaS function). Especially serverless architectures are more decentralized and distributed and make more intentional use of independently provided services. Furthermore, service orchestration logic is shifted to end devices outside the direct scope of the service provisioning system.

Thus, new architecture styles like microservice and serverless architectures might dominate service computing. Furthermore, a resurgence of interest in decentralized approaches known from $\mathrm{P} 2 \mathrm{P}$ related research is observable. That is astonishing because, with the rise of cloud computing (and its centralized service provisioning concept), the research interest in peer-to-peer based approaches 
(and its decentralization philosophy) decreased. However, this seems to change and might be an indicator where cloud computing could be heading in the future. Baldini et al. [42] asked the interesting question, whether serverless extend beyond traditional cloud platforms. If we are looking at the trends investigated in Section 6.2, this seems likely. Modern cloud applications might lose clear boundaries and could evolve into something that could be named service-meshes. Such service-meshes would be composed of small and fine-grained services provided by different and independent providers. Moreover, mobile and edge devices not explicitly belonging to the service provisioning system anymore do the service composition and orchestration. This path might have already started with FaaS and serverless architectures. This forecast might sound astonishing familiar. In the 1960s, the Internet was designed to be decentralized and distributed.

Funding: This research was funded by the German Federal Ministry of Education and Research under Grant No. 13FH021PX4 (Project CloudTRANSIT).

Acknowledgments: I would like to thank Peter-Christian Quint, (Lübeck University of Applied Sciences, Germany), Dirk Reimers (buchhalter.pro GmbH, Lübeck, Germany), Derek Palme (fat IT solutions GmbH, Kiel, Germany), Thomas Finnern (wilhelm.Tel GmbH, Stadtwerke Norderstedt, Germany), René Peinl (Hof University of Applied Sciences, Germany), Bob Duncan (University of Aberdeen, UK), Magnus Westerlund (Arcada University of Applied Sciences, Helsinki, Finland), and Josef Adersberger (QAWare GmbH, Munich, Germany) for their direct or indirect contributions to our research. Without their hard work, their inspiring ideas, their practitioner awareness, or their outside-the-box-thinking, this contribution would not have been possible.

Conflicts of Interest: The author declares no conflict of interest. The founding sponsor had no role in the design of the study; in the collection, analyses, or interpretation of data; in the writing of the manuscript, and in the decision to publish the results.

\section{Abbreviations}

The following abbreviations are used in this manuscript:

$\begin{array}{ll}\text { AMQP } & \text { Advanced Message Queuing Protocol } \\ \text { API } & \text { Application Programming Interface } \\ \text { GCE } & \text { Google Compute Engine } \\ \text { CDMI } & \text { Cloud Data Management Interface } \\ \text { CIMI } & \text { Cloud Infrastructure Management Interface } \\ \text { CNA } & \text { Cloud-Native Application } \\ \text { DLT } & \text { Distributed Ledger Technology (aka blockchain) } \\ \text { IaaS } & \text { Infrastructure as a Service } \\ \text { IoT } & \text { Internet of Things } \\ \text { FaaS } & \text { Function as a Service } \\ \text { HTTP } & \text { Hypertext Transfer Protocol } \\ \text { NIST } & \text { National Institute of Standards and Technology } \\ \text { OCI } & \text { Open Container Initiative } \\ \text { OCCI } & \text { Open Cloud Computing Interface } \\ \text { OVF } & \text { Open Virtualization Format } \\ \text { OS } & \text { Operating System } \\ \text { P2P } & \text { Peer-to-Peer } \\ \text { PaaS } & \text { Platform as a Service } \\ \text { QoS } & \text { Quality of Service } \\ \text { REST } & \text { Representational State Transfer } \\ \text { SaaS } & \text { Software as a Service } \\ \text { SOA } & \text { Service-Oriented Architecture } \\ \text { SOC } & \text { Service-Oriented Computing } \\ \text { TOSCA } & \text { Topology and Orchestration Specification for Cloud Applications } \\ \text { UCAML } & \text { Unified Cloud Application Modeling Language } \\ \text { VM } & \text { Virtual Machine } \\ \text { WS-BPEL } & \text { Web Service-Business Process Execution Language } \\ & \end{array}$




\section{References}

1. Weinmann, J. Mathematical Proof if the Inevitability of Cloud Computing, 2011. Available online: http:/ /joeweinman.com/Resources/Joe_Weinman_Inevitability_Of_Cloud.pdf (accessed on 10 July 2018).

2. Mell, P.M.; Grance, T. The NIST Definition of Cloud Computing; Technical Report; National Institute of Standards \& Technology: Gaithersburg, MD, USA, 2011.

3. Kratzke, N.; Quint, P.C. Preliminary Technical Report of Project CloudTRANSIT-Transfer Cloud-Native Applications at Runtime; Technical Report; Preliminary Technical Report; Lübeck University of Applied Sciences: Lübeck, Germany, 2018.

4. Kratzke, N.; Quint, P.C. Understanding Cloud-native Applications after 10 Years of Cloud Computing-A Systematic Mapping Study. J. Syst. Softw. 2017, 126, 1-16, doi:10.1016/j.jss.2017.01.001. [CrossRef]

5. Kratzke, N.; Peinl, R. ClouNS-A Cloud-Native Application Reference Model for Enterprise Architects. In Proceedings of the 2016 IEEE 20th International Enterprise Distributed Object Computing Workshop (EDOCW), Vienna, Austria, 5-9 September 2016; pp. 1-10, doi:10.1109/EDOCW.2016.7584353. [CrossRef]

6. Kratzke, N. Smuggling Multi-Cloud Support into Cloud-native Applications using Elastic Container Platforms. In Proceedings of the 7th International Conference on Cloud Computing and Services Science (CLOSER 2017), Porto, Portugal, 24-26 April 2017; pp. 29-42.

7. Kratzke, N. About the Complexity to Transfer Cloud Applications at Runtime and how Container Platforms can Contribute? In Cloud Computing and Services Science (Revised Selected Papers); Helfert, M., Ferguson, D., Munoz, V.M., Cardoso, J., Eds.; Communications in Computer and Information Science (CCIS); Springer: Berlin, Germany, 2018.

8. Quint, P.C.; Kratzke, N. Towards a Description of Elastic Cloud-native Applications for Transferable Multi-Cloud-Deployments. In Proceedings of the 1st International Forum on Microservices Odense, Denmark, 25-26 October 2017.

9. Quint, P.C.; Kratzke, N. Towards a Lightweight Multi-Cloud DSL for Elastic and Transferable Cloud-native Applications. In Proceedings of the 8th International Conference on Cloud Computing and Services Science Madeira, Portugal, 19-21 March 2018.

10. Aderaldo, C.M.; Mendonça, N.C.; Pahl, C.; Jamshidi, P. Benchmark Requirements for Microservices Architecture Research. In Proceedings of the 1st International Workshop on Establishing the Community-Wide Infrastructure for Architecture-Based Software Engineering, Buenos Aires, Argentina, 20-28 May 2017; IEEE Press: Piscataway, NJ, USA, 2017; pp. 8-13, doi:10.1109/ECASE.2017..4. [CrossRef]

11. OASIS. Advanced Message Queueing Protocol (AQMP), version 1.0; OASIS: Manchester, UK, 2012.

12. Kratzke, N. Lightweight Virtualization Cluster-Howto overcome Cloud Vendor Lock-in. J. Comput. Commun. 2014, 2, doi:10.4236/jcc.2014.212001. [CrossRef]

13. Kratzke, N.; Quint, P.C.; Palme, D.; Reimers, D. Project Cloud TRANSIT-Or to Simplify Cloud-native Application Provisioning for SMEs by Integrating Already Available Container Technologies. In European Project Space on Smart Systems, Big Data, Future Internet-Towards Serving the Grand Societal Challenges; Kantere, V., Koch, B., Eds.; SciTePress: Setubal, Portugal, 2016.

14. Hogan, M.; Fang, L.; Sokol, A.; Tong, J. Cloud Infrastructure Management Interface (CIMI) Model and RESTful HTTP-Based Protocol; Version 2.0.0c; Distributed Management Task Force, 2015. Available online: https://www.dmtf.org/sites/default/files/standards/documents/DSP0263_1.0.1.pdf (accessed on 13 August 2018).

15. Nyren, R.; Edmonds, A.; Papaspyrou, A.; Metsch, T. Open Cloud Computing Interface (OCCI)—Core; Version 1.2; Open Grid Forum, 2016. Available online: https:/ / www.ogf.org/documents/GFD.221.pdf (accessed on 13 August 2018).

16. Metsch, T.; Edmonds, A. Open Cloud Computing Interface (OCCI)_Infrastructure; Version 1.2; Open Grid Forum. 2016. Available online: https://www.ogf.org/documents/GFD.224.pdf (accessed on 13 August 2018).

17. SNIA. Cloud Data Management Interface (CDMI); Version 1.1.1; SNIA, 2016. Available online: https: //www.snia.org/sites/default/files/CDMI_Spec_v1.1.1.pdf (accessed on 13 August 2018).

18. System Virtualization, Partitioning, and Clustering Working Group. Open Virtualization Format Specification; Version 2.1.1; Distributed Management Task Force, 2015. Available online: https:/ /www.dmtf.org/sites / default/files/standards/documents/DSP0243_2.1.1.pdf (accessed on 13 August 2018). 
19. OCI. Open Container Initiative. 2015. Available online: https://github.com/opencontainers/runtime-spec (accessed on 13 August 2018).

20. OASIS. Topology and Orchestration Specification for Cloud Applications (TOSCA), version 1.0; OASIS, 2013. Available online: http:/ / docs.oasis-open.org/tosca/TOSCA/v1.0/os/TOSCA-v1.0-os.pdf (accessed on 13 August 2018).

21. Opara-Martins, J.; Sahandi, R.; Tian, F. Critical review of vendor lock-in and its impact on adoption of cloud computing. In Proceedings of the International Conference on Information Society (i-Society 2014), London, UK, 10-12 November 2014; pp. 92-97, doi:10.1109/i-Society.2014.7009018. [CrossRef]

22. Ashtikar, S.; Barker, C.; Clem, B.; Fichadia, P.; Krupin, V.; Louie, K.; Malhotra, G.; Nielsen, D.; Simpson, N.; Spence, C. Open Data Center Alliance Best Practices: Architecting Cloud-Aware Applications Rev. 1.0; Technical Report; Open Data Center Alliance, 2014. Available online: https:/ / oaca-project.github.io/ files / Architecting\%20Cloud-Aware\%20Applications\%20Best\%20Practices\%20Rev\%201.0.pdf (accessed on 13 August 2018).

23. Bohn, R.B.; Messina, J.; Liu, F.; Tong, J.; Mao, J. NIST Cloud Computing Reference Architecture. In World Congress on Services (SERVICES 2011); IEEE Computer Society: Washington, DC, USA, 2011; pp. 594-596, doi:10.1109/SERVICES.2011.105.

24. Quint, P.C.; Kratzke, N. Overcome Vendor Lock-In by Integrating Already Available Container Technologies-Towards Transferability in Cloud Computing for SMEs. In Proceedings of the 7th International Conference on Cloud Computing, GRIDS and Virtualization, Rome, Italy, 20-24 March 2016.

25. Ardagna, D.; Casale, G.; Ciavotta, M.; Pérez, J.F.; Wang, W. Quality-of-service in cloud computing: Modeling techniques and their applications. J. Internet Serv. Appl. 2014, 5, 11, doi:10.1186/s13174-014-0011-3. [CrossRef]

26. White, G.; Nallur, V.; Clarke, S. Quality of service approaches in IoT: A systematic mapping. J. Syst. Softw. 2017, 132, 186-203. [CrossRef]

27. Newman, S. Building Microservices; O’Reilly Media: Sebastopol, CA, USA, 2015.

28. Namiot, D.; Sneps-Sneppe, M. On micro-services architecture. Int. J. Open Inf. Technol. 2014, 2, $24-27$.

29. Cerny, T.; Donahoo, M.J.; Pechanec, J. Disambiguation and Comparison of SOA, Microservices and Self-Contained Systems. In Proceedings of the International Conference on Research in Adaptive and Convergent Systems-RACS '17, Krakow, Poland, 20-23 September 2017, doi:10.1145/3129676.3129682. [CrossRef]

30. Taibi, D.; Lenarduzzi, V.; Pahl, C. Architectural Patterns for Microservices: A Systematic Mapping Study. In Proceedings of the 8th International Conference on Cloud Computing and Services Science (CLOSER'18), Madeira, Portugal, 19-21 March 2018.

31. Jamshidi, P.; Pahl, C.; Mendonça, N.C.; Lewis, J.; Stefan Tilkov, T. Microservices The Journey So Far and Challenges Ahead. IEEE Softw. 2018, 35, 24-35. [CrossRef]

32. Balalaie, A.; Heydarnoori, A.; Jamshidi, P. Microservices Architecture Enables DevOps: Migration to a Cloud-Native Architecture. IEEE Softw. 2016, doi:10.1109/MS.2016.64. [CrossRef]

33. Jabbari, R.; bin Ali, N.; Petersen, K.; Tanveer, B. What is DevOps? A Systematic Mapping Study on Definitions and Practices. In Proceedings of the Scientific Workshop Proceedings of XP2016, Scotland, UK, 24 May 2016, doi:10.1145/2962695.2962707. [CrossRef]

34. Bergmayr, A.; Breitenbücher, U.; Ferry, N.; Rossini, A.; Solberg, A.; Wimmer, M.; Kappel, G.; Leymann, F. A Systematic Review of Cloud Modeling Languages. ACM Comput. Surv. 2018, 51, 39, doi:10.1145/3150227. [CrossRef]

35. Adam Wiggins. The Twelve-Factor App. 2014. Available online: https://12factor.net (accessed on 13 August 2018).

36. Martin Fowler. Circuit Breaker. 2014. Available online: https://martinfowler.com/bliki/CircuitBreaker. html (accessed on 11 August 2018).

37. Fehling, C.; Leymann, F.; Retter, R.; Schupeck, W.; Arbitter, P. Cloud Computing Patterns; Springer: Berlin, Germany, 2014.

38. Erl, T.; Cope, R.; Naserpour, A. Cloud Computing Design Patterns; Springer: Berlin, Germany, 2015. 
39. Verma, A.; Pedrosa, L.; Korupolu, M.; Oppenheimer, D.; Tune, E.; Wilkes, J. Large-scale cluster management at Google with Borg. In Proceedings of the Tenth European Conference on Computer Systems-EuroSys'15, Zhangiiajie, China, 18-20 November 2015; pp. 1-17, doi:10.1145/2741948.2741964. [CrossRef]

40. Michael Roberts and John Chapin. What Is Serverless? O'Reilly: Sebastopol, CA, USA, 2016.

41. Baldini, I.; Cheng, P.; Fink, S.J.; Mitchell, N.; Muthusamy, V.; Rabbah, R.; Suter, P.; Tardieu, O. The serverless trilemma: Function composition for serverless computing. In Proceedings of the 2017 ACM SIGPLAN International Symposium on New Ideas, New Paradigms, and Reflections on Programming and Software, Vancouver, BC, Canada, 25-27 October 2017, doi:10.1145/3133850.3133855. [CrossRef]

42. Baldini, I.; Castro, P.; Chang, K.; Cheng, P.; Fink, S.; Ishakian, V.; Mitchell, N.; Muthusamy, V.; Rabbah, R.; Slominski, A.; et al. Serverless Computing: Current Trends and Open Problems. In Research Advances in Cloud Computing; Springer: Singapore, 2017; pp. 1-20, doi:10.1007/978-981-10-5026-8_1.

43. Martin Fowler. Microservices-A Definition of this new Architectural Term. 2014. Available online: https://martinfowler.com/articles/microservices.html (accessed on 13 August 2018).

44. Villamizar, M.; Garcés, O.; Ochoa, L.; Castro, H.; Salamanca, L.; Verano, M.; Casallas, R.; Gil, S.; Valencia, C.; Zambrano, A.; et al. Cost comparison of running web applications in the cloud using monolithic, microservice, and AWS Lambda architectures. Serv. Oriented Comput. Appl. 2017, doi:10.1007/s11761-017-0208-y. [CrossRef]

45. Dragoni, N.; Giallorenzo, S.; Lafuente, A.L.; Mazzara, M.; Montesi, F.; Mustafin, R.; Safina, L. Microservices: Yesterday, Today, and Tomorrow. In Present and Ulterior Software Engineering; Mazzara, M., Meyer, B., Eds.; Springer International Publishing: Cham, Switzerland, 2017; pp. 195-216, doi:10.1007/978-3-319-67425-4_12.

46. Pahl, C.; Brogi, A.; Soldani, J.; Jamshidi, P. Cloud Container Technologies: A State-of-the-Art Review. IEEE Trans. Cloud Comput. 2017, 1, doi:10.1109/TCC.2017.2702586. [CrossRef]

47. Hindman, B.; Konwinski, A.; Zaharia, M.; Ghodsi, A.; Joseph, A.D.; Katz, R.; Shenker, S.; Stoica, I. Mesos: A Platform for Fine-grained Resource Sharing in the Data Center. In Proceedings of the 8th USENIX Conference on Networked Systems Design and Implementation, Boston, MA, USA, 30 March-1 April 2011; USENIX Association: Berkeley, CA, USA, 2011; pp. 295-308.

48. Pérez, A.; Moltó, G.; Caballer, M.; Calatrava, A. Serverless computing for container-based architectures. Future Gener. Comput. Syst. 2018, 83, 50-59. [CrossRef]

49. Baldini, I.; Castro, P.; Cheng, P.; Fink, S.; Ishakian, V.; Mitchell, N.; Muthusamy, V.; Rabbah, R.; Suter, P. Cloud-native, event-based programming for mobile applications. In Proceedings of the International Conference on Mobile Software Engineering and Systems, Austin, TX, USA, 16-17 May 2016; pp. 287-288.

50. Cozzolino, V.; Ding, A.Y.; Ott, J. FADES: Fine-grained edge offloading with unikernels. In Proceedings of the Workshop on Hot Topics in Container Networking and Networked Systems, Los Angeles, CA, USA, 25 August 2017; pp. 36-41.

51. Koller, R.; Williams, D. Will Serverless End the Dominance of Linux in the Cloud? In Proceedings of the 16th Workshop on Hot Topics in Operating Systems, Whistler, BC, Canada, 8-10 May 2017; pp. 169-173.

52. Bratterud, A.; Happe, A.; Duncan, R.A.K. Enhancing cloud security and privacy: The Unikernel solution. In Proceedings of the 8th International Conference on Cloud Computing, GRIDs, and Virtualization, Athens, Greece, 19-23 February 2017.

53. Happe, A.; Duncan, B.; Bratterud, A. Unikernels for Cloud Architectures: How Single Responsibility Can Reduce Complexity, Thus Improving Enterprise Cloud Security; SciTePress: Setubal, Portugal, 2017; Volume 2016; pp. $1-8$.

54. Duncan, B.; Happe, A.; Bratterud, A. Cloud Cyber Security: Finding an Effective Approach with Unikernels. In Security in Computing and Communications; IntechOpen: London, UK, 2017; p. 31.

55. Compastié, M.; Badonnel, R.; Festor, O.; He, R.; Lahlou, M.K. Unikernel-based Approach for Software-Defined Security in Cloud Infrastructures. In Proceedings of the NOMS 2018-IEEE/IFIP Network Operations and Management Symposium, Taipei, Taiwan, 23-27 April 2018.

56. Rahimi, M.R.; Ren, J.; Liu, C.H.; Vasilakos, A.V.; Venkatasubramanian, N. Mobile Cloud Computing: A Survey, State of Art and Future Directions. MONET 2014, 19, 133-143. [CrossRef]

57. Pooranian, Z.; Abawajy, J.H.; P, V.; Conti, M. Scheduling Distributed Energy Resource Operation and Daily Power Consumption for a Smart Building to Optimize Economic and Environmental Parameters. Energies 2018, 11, 1348. [CrossRef] 
58. Pooranian, Z.; Chen, K.C.; Yu, C.M.; Conti, M. RARE: Defeating side channels based on data-deduplication in cloud storage. In Proceedings of the IEEE INFOCOM 2018-IEEE Conference on Computer Communications Workshops (INFOCOM WKSHPS), Honolulu, HI, USA, 15-19 April 2018; pp. 444-449, doi:10.1109/INFCOMW.2018.8406888. [CrossRef]

59. Liu, M.; Koskela, T.; Ou, Z.; Zhou, J.; Riekki, J.; Ylianttila, M. Super-peer-based coordinated service provision. Advanced Topics in Cloud Computing. J. Netw. Comput. Appl. 2011, 34, 1210-1224. [CrossRef]

60. Westerlund, M.; Kratzke, N. Towards Distributed Clouds-A review about the evolution of centralized cloud computing, distributed ledger technologies, and a foresight on unifying opportunities and security implications. In Proceedings of the 16th International Conference on High Performance Computing and Simulation (HPCS 2018), Orléans, France, 16-20 July 2018.

61. Kaur, T.; Chana, I. Energy Efficiency Techniques in Cloud Computing: A Survey and Taxonomy. ACM Comput. Surv. 2015, 48, 22:1-22:46, doi:10.1145/2742488. [CrossRef]

62. Tosatto, A.; Ruiu, P.; Attanasio, A. Container-Based Orchestration in Cloud: State of the Art and Challenges. In Proceedings of the 2015 Ninth International Conference on Complex, Intelligent, and Software Intensive Systems, Blumenau, Brazil, 8-10 July 2015; pp. 70-75, doi:10.1109/CISIS.2015.35. [CrossRef]

63. Peinl, R.; Holzschuher, F.; Pfitzer, F. Docker Cluster Management for the Cloud-Survey Results and Own Solution. J. Grid Comput. 2016, 14, 265-282. [CrossRef]

64. Spillner, J. Practical Tooling for Serverless Computing. In Proceedings of the10th International Conference on Utility and Cloud Computing, Austin, TX, USA, 5-8 December 2017; ACM: New York, NY, USA, 2017; pp. 185-186, doi:10.1145/3147213.3149452. [CrossRef]

65. Lynn, T.; Rosati, P.; Lejeune, A.; Emeakaroha, V. A Preliminary Review of Enterprise Serverless Cloud Computing (Function-as-a-Service) Platforms. In Proceedings of the 2017 IEEE International Conference on Cloud Computing Technology and Science (CloudCom), Hong Kong, China, 11-14 December 2017; pp. 162-169, doi:10.1109/CloudCom.2017.15. [CrossRef]

66. Van Eyk, E.; Toader, L.; Talluri, S.; Versluis, L.; Uta, A.; Iosup, A. Serverless is More: From PaaS to Present Cloud Computing. Sep/Oct issue. IEEE Internet Comput. 2018, 22. Available online: https: / / atlarge-research.com/pdfs/serverless-history-now-future18ieeeic.pdf (accessed on 13 August 2018).

67. Van Eyk, E.; Iosup, A.; Abad, C.L.; Grohmann, J.; Eismann, S. A SPEC RG Cloud Group's Vision on the Performance Challenges of FaaS Cloud Architectures. In Proceedings of the 8th ACM/SPEC on International Conference on Performance Engineering, ICPE 2018, Berlin, Germany, 9-13 April 2018.

68. Ylianttila, M.; Riekki, J.; Zhou, J.; Athukorala, K.; Gilman, E. Cloud Architecture for Dynamic Service Composition. Int. J. Grid High Perform. Comput. 2012, 4, 17-31, doi:10.4018/jghpc.2012040102. [CrossRef]

69. Zhou, J.; Riekki, J.; Sun, J. Pervasive Service Computing toward Accommodating Service Coordination and Collaboration. In Proceedings of the 2009 4th International Conference on Frontier of Computer Science and Technology, Shanghai, China, 17-19 December 2009; pp. 686-691, doi:10.1109/FCST.2009.39. [CrossRef]

70. Huhns, M.N.; Singh, M.P. Service-Oriented Computing: Key Concepts and Principles. IEEE Internet Comput. 2005, 9, 75-81. [CrossRef]

71. Dustdar, S.; Schreiner, W. A survey on web services composition. Int. J. Web Grid Serv. 2005, 1, 1-30. [CrossRef]

72. Papazoglou, M.P.; Traverso, P.; Dustdar, S.; Leymann, F. Service-Oriented Computing: State of the Art and Research Challenges. Computer 2007, 40, 38-45, doi:10.1109/MC.2007.400. [CrossRef]

73. Papazoglou, M.P.; van den Heuvel, W.J. Service oriented architectures: Approaches, technologies and research issues. VLDB J. 2007, 16, 389-415, doi:10.1007/s00778-007-0044-3. [CrossRef]

74. Razavian, M.; Lago, P. A Survey of SOA Migration in Industry; Springer: Berlin, Germany, 2011.

(C) 2018 by the authors. Licensee MDPI, Basel, Switzerland. This article is an open access article distributed under the terms and conditions of the Creative Commons Attribution (CC BY) license (http://creativecommons.org/licenses/by/4.0/). 